\title{
The prototypes of nanozyme-based nanorobots
}

\author{
Jiaying Xie ${ }^{1}$, Yiliang Jin ${ }^{1,2}$, Kelong Fan ${ }^{1,3 凶}$, Xiyun Yan ${ }^{1,3 \bowtie}$ \\ 1 CAS Engineering Laboratory for Nanozyme, Key Laboratory of Protein and Peptide Pharmaceutical, Institute of \\ Biophysics, Chinese Academy of Sciences, Beijing 100101, China \\ 2 School of Future Technology, University of Chinese Academy of Sciences, Beijing 101408, China \\ 3 Academy of Medical Sciences, Zhengzhou University, Zhengzhou 450052, China
}

Received: 28 June 2020 / Accepted: 14 October 2020 / Published online: 20 November 2020

\begin{abstract}
Artificial nanorobot is a type of robots designed for executing complex tasks at nanoscale. The nanorobot system is typically consisted of four systems, including logic control, driving, sensing and functioning. Considering the subtle structure and complex functionality of nanorobot, the manufacture of nanorobots requires designable, controllable and multi-functional nanomaterials. Here, we propose that nanozyme is a promising candidate for fabricating nanorobots due to its unique properties, including flexible designs, controllable enzyme-like activities, and nano-sized physicochemical characters. Nanozymes may participate in one system or even combine several systems of nanorobots. In this review, we summarize the advances on nanozyme-based systems for fabricating nanorobots, and prospect the future directions of nanozyme for constructing nanorobots. We hope that the unique properties of nanozymes will provide novel ideas for designing and fabricating nanorobotics.
\end{abstract}

Keywords Nanorobot, Nanozyme, Logic gate, Nanomotor, Motion control

\section{INTRODUCTION}

The biomolecules created by nature are able to manipulate subtle objects at nano/micro scales, which can be considered as most ideal nanorobots existing in the world. Proteins utilize ATP to perform sophisticated functions in cells, some of which even assemble into supermolecules to finish more complex tasks perfectly. Still, the proteins have never stopped evolving since the day they were created by nature. For decades, scientists have been working on masses of researches to figure out the basic characteristics of those biomolecules; however, there are still many questions left. Nowadays, inspired by these natural nanorobots, scientists are making an endeavor to follow the intelligence of nature by building artificial nanorobots.

Jiaying Xie and Yiliang Jin have contributed equally to this work.

$\triangle$ Correspondence: fankelong@ibp.ac.cn (K. Fan), yanxy@ibp.ac.cn (X. Yan)
Artificial nanorobots, the robots working at nanoscale, have been through tremendous development in recent years. Nanorobotics mainly focuses on two areas: one is the macroscale robots manipulating nanoscale materials; the other is the robots built at nanoscale (Sierra et al. 2005). The latter is capable of performing complex tasks at nanoscale, for example, manipulating atoms or molecules. Nanorobots may be programmed with specific tasks to automatically respond to the changing environment and switch their programs to execute corresponding functions (Loukanov et al. 2019a).

Building a nanorobot is much like the manufacture of a macroscale robot, while the difference is that a nanorobot needs more elaborate manipulation at nanoscale. Similar to the macroscale robots, the primary components needed for nanorobots include a logic control system, a sensing system, a driving system and a functioning system. When the logic control system receives information data from the environment, it operates logically based on the programmed reference 
and output message to other systems. Subsequently, other systems will instruct the nanorobot to finish the tasks, such as the motion control of the driving system or the switch of the function system. At the same time, the sensing system interacts with the environment and provides feedback to the logic control system, thus helping the nanorobot work properly (Celikten and Cetin 2016). These systems of nanorobots are similar to those of macroscale robots, however, building these systems at nanoscale is not as easy as macroscale. Besides the difficulties of assembling materials into an intact structure at nanoscale, the nano effect is also a big challenge, which will change the characteristics of materials at nanoscale. Therefore, more elaborate manipulations should be made to build advanced nanorobots. In the last decade, scientists have made great progress in this field. Among them, nanozyme (nanomaterials with intrinsic enzyme-like activities) may make a great contribution to nanorobotics.

Since the discovery of nanozyme in 2007 (Gao et al. 2007), it is very inspiring that researches on this subject increase explosively all over the world. Materials at nanoscale with intrinsic enzyme-like activities have been used in various areas, especially biomedical applications including disease theranostics (Zhang et al. 2020). Moreover, nanozymes possess advantages of higher stability, easier modification and lower manufacturing cost. Furthermore, as nanomaterials, the inherent physical and chemical properties of nanozymes provide a multimodal platform for manipulating nanorobots (Jiang et al. 2019b).

Is there any chance that nanozymes can make a difference in the fabrication of nanorobots? Indeed, nanozymes are able to help build the logic control system, sensing system, driving system, and functioning system of nanorobots. In this review, we will summarize the recent researches that fabricated nanorobots with nanozymes in different systems.

\section{ELEMENTS OF NANOROBOTS}

As we mentioned above, nanorobots are composed of several primary systems, while in some cases they are divided into certain nanomachines with incomplete systems.

In numerous reports, nanomachines and nanorobots are usually used as synonyms. In fact, nanomachines may be considered as a step of building nanorobots. Nanomachines consist of only one or a few systems of nanorobots, and therefore they may not be fully functional as that of nanorobots (Loukanov et al. 2019a). Nanomachines are partial components for the fabrication of nanorobots, and we are able to build a whole nanorobot by putting them together. Nanomachines have been studied widely and scientists already created nanomotors, multifunctional nanoparticles, nano-biosensors, nanogears, nanojoints and other nanostructures (Yang et al. 2018). These nanodevices may not contain the entire systems of nanorobots. For example, some nanoparticles lack the driving system, and many nanomachines do not include the logic control system. In this review, some of the nanodevices we mentioned as follows belong to nanomachines.

\section{Logic control system}

Electronic information industry endows our daily life with astonishing convenience and progress, largely depending on Boolean algebra. Boolean algebra can be applied to any type of information expressed as 0 and 1 , which is the fundamental operation for computers (Tregubov et al. 2018). Therefore, a robot at nanoscale also needs to be built on Boolean algebra. The input signal 0 or 1 are processed at the logic control system, after which the output signal 0 or 1 will be given to other systems, so that the latter can take an operation. Furthermore, there is another important component for the input information-the logic gate. As same as the computer language, the gate is capable of processing the input signal then the computed information will be transmitted to the control center, which may be the unary YES and NOT gates, as well as the binary AND and OR gates. These logic gates have been built by various nanomaterials and work in a number of entities at nanoscale.

Nanomaterials like DNA, protein and other molecules are appropriate for the fabrication of logic gates. Constructed by DNA strands, the nucleic acid-based logic gate could control AND and OR functions through modified fluorescence (Yoshida and Yokobayashi 2007). When the ligands were added to the system and matched with the corresponding aptamers, the fluorescence of corresponding ON (1) or OFF (0) information would be outputted (Fig. 1). For the AND gate, if both of the ligands were matched (the input signals are " 1 " and " 1 "), the change of fluorescence would transmit ON (1) as output, otherwise the output information would be OFF (0). The OR gate was different from the AND gate, that is, as long as one of the ligands were matched (input signals are " 1 " and " 0 ", "0" and " 1 ", "1" and " 1 "), the output would be ON (1). As we can see, the key point of logic gate is the threshold conditions for ON and OFF signal, and how to set the threshold depends on the materials and the way they were built. 


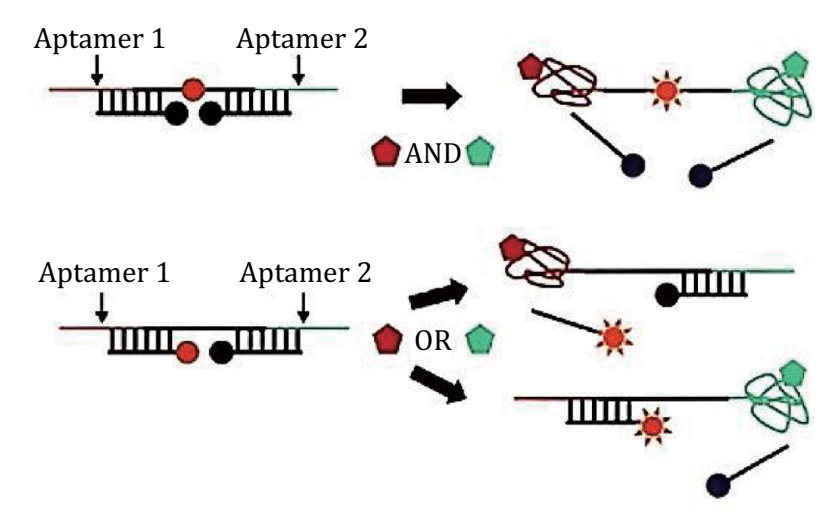

Fig. 1 The working model of "AND" and "OR" gates of a DNAbased logic gate. Because of the quencher moieties, attenuated fluorescence means the "OFF" state, and enhancement of fluorescence means the "ON" state. Reproduced with permission of Royal Society of Chemistry (Yoshida and Yokobayashi 2007)

\section{Driving system-nanomotor}

As a nanomachine, nanomotor is driven by power instead of doing Brownian motion. Many kinds of micro/nanomotors have already been developed, such as nanowires (Guo et al. 2018; Wang et al. 2017a), helical motors (Li et al. 2014; Manesh et al. 2013), Janus particles (Ma et al. 2016; Qin et al. 2017), micro/nanorockets (micro/nanotubular) (Gao et al. 2012; Xu et al. 2018; Zha et al. 2018), etc. (Fig. 2). Some nanomotors obtain energy from external devices and convert it to motive power, meanwhile others catalyze fuels in the environment to move against the Brownian motion and other barriers. Therefore, nanomotors can be classified as fuel-free nanomotors and fuel-dependent nanomotors.

The fuel-free nanomotor receives energy from an external source such as magnetic, electric, acoustic and light fields, after which the driving system will convert the energy to power the nanomotor so that the nanorobot can overcome the obstacle of Brownian motion or resistance in fluid (Wang and Pumera 2015; Wang et al. 2019). While the fuel-dependent nanomotor does not need energy from an external source, it utilizes the nonbiotoxic or biocompatible fuels in the environment. So far, the diverse fuels that have been reported include $\mathrm{H}_{2} \mathrm{O}_{2}$, $\mathrm{Br}_{2}$, or $\mathrm{I}_{2}$ solutions, hydrazine, acidic and alkaline solutions, and even methanol, diazomethane, azides, and glucose oxides (Mano and Heller 2005; Mirkovic et al. 2010; Wang and Pumera 2015).

A large part of micro/nanomotors are designed for applications in living bodies, in particular with biomedical platforms (Gao et al. 2018; Wang et al. 2019). It is reported that excess $\mathrm{H}_{2} \mathrm{O}_{2}$ accumulated in tumor sites (Szatrowski and Nathan 1991), which provides favorable conditions for $\mathrm{H}_{2} \mathrm{O}_{2}$-dependent nanomotors in cancer therapy. Moreover, tumor sites also show an acidic pH level (Vaupel 2004), which is partially caused by the elevated level of lactic acid (Kim and Dang 2006). Guix et al. built a $\mathrm{CaCO}_{3}$-based Janus particle to help deliver drugs for cancer cells by utilizing the acidic environment (Guix et al. 2016). The Janus particle, a hemispherical nanomotor decorated by $\mathrm{CaCO}_{3}$ would generate $\mathrm{CO}_{2}$ bubbles to power the motor in acidic environment, then the continuous reaction would dissolve the $\mathrm{CaCO}_{3}$ shell leading to the release of drugs at the tumor site.

Furthermore, it is also important to control the pattern of motion, which means modulating the moving direction, speed, starting or stopping of the movement. To exert the control, we may search for solutions like external wireless influence or inner self modulation. Various methods have been applied to the motion control of the nanomotors, such as magnetic guidance, thermally control, chemical response, phototaxis, particle communication, etc. (Hong et al. 2010; Wang and Pumera 2015; Wang and Manesh 2010).

\section{Sensing system}

The sensing system is another crucial system for the operation of nanorobots/nanomachines. It communicates with the environment to obtain information, so that the control system acts upon these received data to modulate other systems. Nanomachines without control system may also depend on the sensing system for direct modulation. It may have an intimate connection with driving system and influence the motion, or it can switch the activation and inhibition of function systems. Zhang et al. built a jellyfish-like micromotor for DNA sensing, the motion of which was controlled by the DNA-dependent sensing system (Zhang et al. 2019). The micromotor was composed of an umbrella-shaped multi-metallic body, a bunch of DNA hybridization and catalase decoration (Fig. 3A). When the target DNA matched with the DNA of sensing units, sensing units disassembled and released power units, reducing the motion speed (Fig. 3B). The sensing system thus could modulate the motion and transmit the information about DNA detection by reducing the speed. In addition, the sensing system built by targeting molecules also affected the function system such as an artificial engineered platelet (Hu et al. 2016). Decorated with targeting molecules on the surface, the drug-encompassed platelet could sense and target myeloma cells via 
A

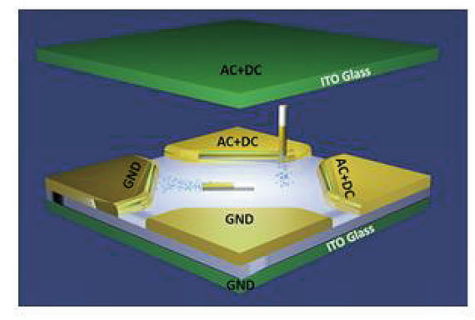

B

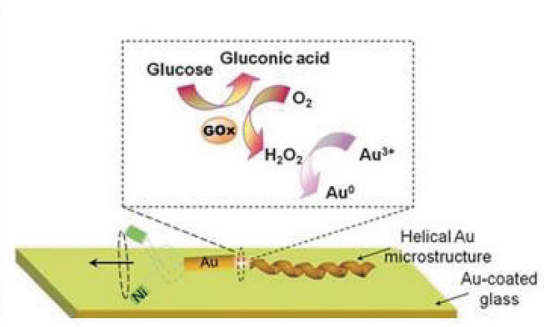

C

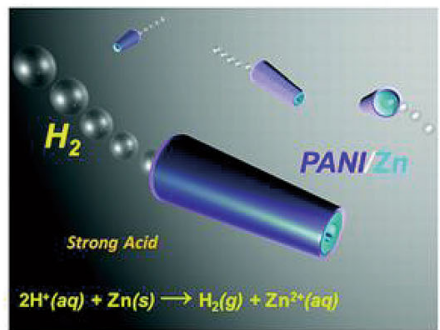

D

Propulsion mechanism

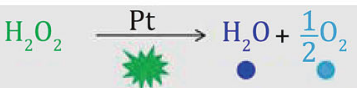

$0.5 \mu \mathrm{m}$

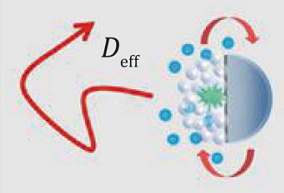

Enhanced diffusion

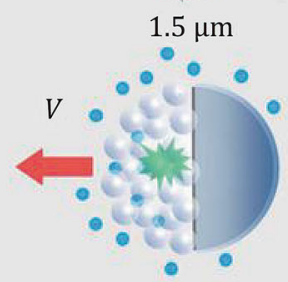

Phoretic motion

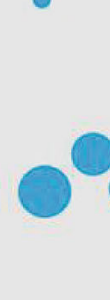

10

Bubble propulsion

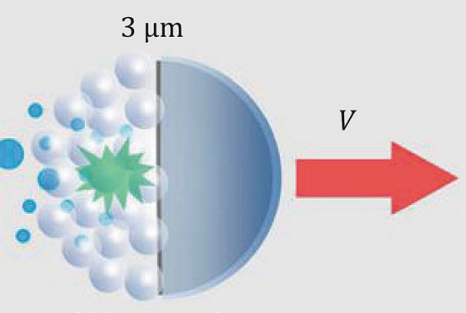

Fig. 2 Different types of micro/nanomotors. A An electric-field-guided catalytic nanomotor. Reproduced with permission of American Chemical Society (Guo et al. 2018). B A magnetically powered enzyme-functionalized helical nanomotor. Reproduced with permission of Royal Society of Chemistry (Manesh et al. 2013). C A Zn nanozyme-based microrocket. Reproduced with permission of American Chemical Society (Gao et al. 2012). D A Pt nanozymes-based Janus particle. Reproduced with permission of American Chemical Society (Ma et al. 2016)

A

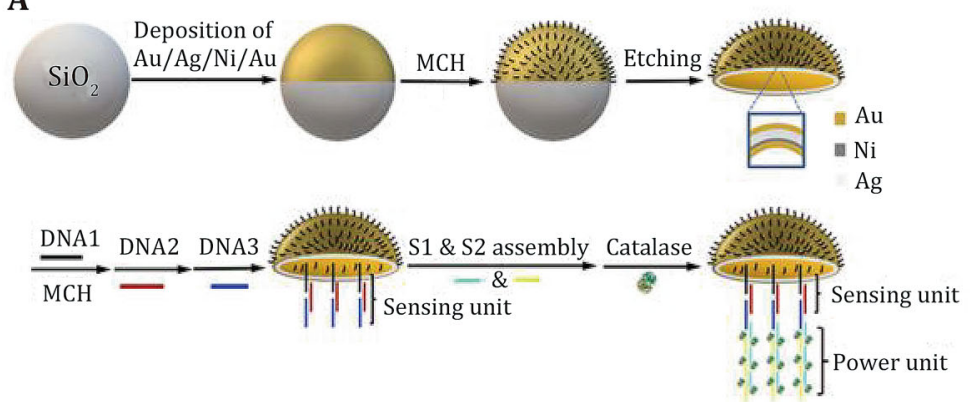

$\mathbf{B}$

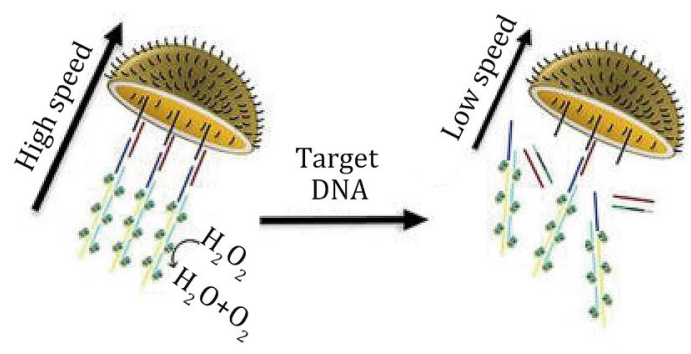

Fig. 3 A Steps of fabricating the jellyfish-like micromotor. B The working mechanism of DNA sensing. Reproduced with permission of American Chemical Society (Zhang et al. 2019)

alendronate and P-selectin. After internalization by myeloma cells, cell death would be induced through the release of bortezomib.

\section{Function system}

The function system is the most vital system, while all the other systems are here to help execute the functions. The final purpose of building nanorobots is to achieve subtle work at nanoscale in various areas, especially medical therapy. There have been many nano/micromotors endowed with medical functions, for instance, cancer-targeted delivery, diagnosis and imaging-guided therapy (Wang et al. 2019). Medical nano/micromotors are capable of delivering drugs to tumor sites like the Janus particle (Guix et al. 2016); or they may depend on the sensing system to detect target molecules for cancer diagnosis, such as the jellyfish-like micromotor for DNA sensing (Zhang et al. 2019). As for imaging-guided 
therapy functions, fluorescent materials enabled nano/ micromotors to monitor in vivo (Wan et al. 2019).

In this area, the nanoparticle is a burgeoning method for medical therapy. Nanoparticles, composed of multiple elements, are capable of performing smart work at nanoscales. In the progress of cancer nanomedicines, nanoparticles have made a remarkable contribution to drug delivery, tumor therapy, in vivo imaging, etc. (Loukanov et al. 2019b). However, many nanoparticles usually lack some systems, for example, the driving system, and make movement just following the environment. As we can see, nanoparticles are a great platform for multiple functions, and these functions need to be integrated into nanomachines or nanorobots, acting like a stack of diverse bricks for building nanomachines. The functions we required determine the applications of these nanomachines or nanorobots.

\section{NANOZYMES AND NANOROBOTS}

Nanozymes possess enzyme-like activities, indicating that the catalytic efficiency and mechanism are similar to that of natural enzymes. Most of the nanozymes follow the Michaelis-Menten kinetics, and there have already been standardized methods to study the catalytic activities of certain nanozymes (Jiang et al. 2018). By the end of May 2020, more than 900 nanomaterials have been reported to show enzymatic activity. Nanomaterials such as $\mathrm{Fe}_{3} \mathrm{O}_{4}, \mathrm{Co}_{3} \mathrm{O}_{4}, \mathrm{CuO}, \mathrm{Au}, \mathrm{Ag}$, Pt and $\mathrm{Pd}$ etc. were found to perform catalytic activities comparable to natural enzymes (Yan and Gao 2020). In addition, those nanozymes possess enzyme-like activities including peroxidase-like activity, oxidase-like activity, catalase-like activity, superoxide dismutase-like activity, sulfite oxidase-like activity, protease-like activity and nuclease-like activity (Gao et al. 2020). Nanozymes may take place of the natural peroxidase or oxidase, even the protease and nuclease in artificial applications.

\section{Enzyme-like activities and other properties}

Peroxidase-like activity is a common property of many nanozymes, and peroxidase-like nanozymes reduce peroxide to generate transient reactive intermediates like free radicals, which further react with another substrate quickly. Catalase-like nanozymes catalyze the decomposition of $\mathrm{H}_{2} \mathrm{O}_{2}$ into molecular oxygen $\left(\mathrm{O}_{2}\right)$ and water $\left(\mathrm{H}_{2} \mathrm{O}\right)$, with oxygen bubbles produced during the reaction. Besides, the oxidase-like nanozymes oxidize substrates in the presence of $\mathrm{O}_{2}$, among which, the glucose oxidase (GOx)-like nanozymes catalyze glucose to generate $\mathrm{H}_{2} \mathrm{O}_{2}$ and gluconic acid. These enzyme-like activities are usually used to treat tumor cells or detect biomolecules in vivo or in vitro.

The oxidoreductase activities often catalyze the colorimetric reaction, the results of which are visualized and quantified. In tumor cells, these reactions play a role in modulating the cellular networks so as to treat cancer. Being biocompatible with low biotoxicity, nanozymes are favorable materials for biomedical applications and environment pollution (Munir et al. 2020). With high stability, low cost, easy large-scale production and simple modification, nanozymes act as a better choice for the substitution of natural enzymes. More importantly, some of them are endowed with several enzyme-like activities and catalyze cascade reactions just by one nanozyme (Fan et al. 2018b; He et al. 2013; $\mathrm{Xu}$ et al. 2019). This is a huge step for enzyme utilization. As natural enzymes, activators and inhibitors control their activities in various ways due to their physicochemical properties. For example, the gold nanozymes can be modulated by metal ions. $\mathrm{Hg}^{2+}$ and $\mathrm{Pb}^{2+}$ may endow $\mathrm{Au}$ nanozymes with the peroxidaselike activity. (Wang et al. 2012).

Apart from the enzyme-like activity, different nanozymes boast diverse physiochemical properties. For instance, iron oxide-based nanomaterials are able to respond to magnetic field, the color of cerium oxide nanozymes change with the states of $\mathrm{Ce}^{3+}$ and $\mathrm{Ce}^{4+}$, and $\mathrm{Au}$ nanozymes are capable of absorbing near infrared (NIR) to generate heat.

\section{How can nanozyme help nanorobots?}

Activities of nanozymes are similar to that of natural enzymes, as activators and inhibitors also have an impact on nanozymes. Thus, it is proper to utilize nanozymes in the logic control system so that the "ON" and "OFF" of logic gate will be modulated by activators and inhibitors, and then transformed to the signal " 0 " and " 1 ". Apart from the logic control system, the driving system is also suitable for these enzyme-like activities. For fuel-dependent nanomotors, enzyme-like activities of catalysts help power the motion, especially the catalase-like activity. In addition, nanozymes can not only catalyze the fuels for power but also control the motion. Decorated with targeting molecules, nanozymes have the potential to be applied in motion control. Some special features make nanozymes a promising material in the driving systems of nanorobots. For example, magnetic nanozymes help control the motion and guide the whole machine to the targeting position. Most importantly, their catalytic activities are powerful tools for the biomedicine applications, which provides multiple choices for the function systems. In conclusion, 
nanozymes show great potential in nanorobots, and their applications will be discussed in detail in the following sections.

\section{Nanozyme-based logic gate for control system}

Signal "0" and "1" are the most important information for the control system as well as the ON and OFF of the logic gates. Therefore, activators and inhibitors function as proper signals for the logic gate and control system through modulating the $\mathrm{ON}$ and $\mathrm{OFF}$ activities of enzymes. There are some enzymes utilized in the logic gating function already, and the substrates and products functioned as the input and output information. With glucose oxidase (GOx), horseradish peroxidase (HRP), and invertase (INV) on the surface, these enzyme-coated carbon nanotubes generated spectroscopic and electrochemical output signals (Kim et al. 2011). Compared with natural enzymes, nanozymes are more easily to be modified with specific properties. Owing to reversiblycontrolled enzyme-like activities, nanozymes are promising logic-gated nanomaterials for their simple instruction in the logic control system, as the exchange of their activity is able to present the signal " 0 " and " 1 ".

\section{Au nanozymes modulated by metal ions}

Metal ions may act as activators and inhibitors. By pairing with the ions in the environment, nanozymebased logic gates are able to transmit output signals. Lien et al. utilized enzyme-like gold nanozyme to construct four types of logic gates combining with five metal ions including $\mathrm{Ag}^{+}, \mathrm{Bi}^{3+}, \mathrm{Pb}^{2+}, \mathrm{Pt}^{4+}$ and $\mathrm{Hg}^{2+}$ (Lien et al. 2013). These metal ions here were activators or inhibitors which could be utilized as input signals whereas the output signals were the color changes of chromogenic substrate induced by the multiple enzymelike activities. For the OR gate which utilized the $\mathrm{Hg}^{2+}$ and $\mathrm{Bi}^{3+}$ as the input signals, the output signals would be 0 (OFF) only in the absence of both ions. Otherwise, either of the two ions would activate the catalase (CAT)like activity of the Au nanozymes and the output signals would be $1(\mathrm{ON})$ (Fig. 4A). Then the AND gate here was controlled by the $\mathrm{Pt}^{4+}$ and $\mathrm{Hg}^{2+}$ ions, and the presence of both $\mathrm{Pt}^{4+}$ and $\mathrm{Hg}^{2+}$ ions endowed the gate with oxidase (OX)-like activity leading to the $1(\mathrm{ON})$ as output signal. Other three situations would make the 0 (OFF) (Fig. 4B). With regard to the INHIBIT gate, it inhibited the peroxidase (POX)-like activity of the $\mathrm{Pb}^{2+}-\mathrm{Au}$ nanozymes by the $\mathrm{Hg}^{2+}$ ions, so that the $\mathrm{Pb}^{2+}-\mathrm{Au}$ NPs without $\mathrm{Hg}^{2+}$ ions would come out 1 (ON) (Fig. 4C). Last, they built a XOR (Exclusive-OR) logic gate via regulation of the peroxidase (POX)-like activity through $\mathrm{Ag}^{+}$and $\mathrm{Bi}^{3+}$ ions. This XOR logic gate could provide the output signals with $1(\mathrm{ON})$ in two situations, only when one of the two ions $\mathrm{Bi}^{3+}$ and $\mathrm{Ag}^{+}$ was deposited on the Au nanozymes (Fig. 4D). If the two ions $\mathrm{Bi}^{3+}$ and $\mathrm{Ag}^{+}$were both added to the gate at the same time, then the output would be 0 (OFF). These four logic gates-" $\mathrm{Hg}^{2+} / \mathrm{Bi}^{3+}(\mathrm{OR})-\mathrm{Au} \quad \mathrm{NP}_{\mathrm{CAT}}$ ", " $\mathrm{Pb}^{4+} /$ $\mathrm{Hg}^{2+}$ (AND)-Au NP${ }_{\mathrm{Ox}}$ ", " $\mathrm{Pb}^{2+} / \mathrm{Hg}^{2+}$ (INHIBIT)-Au NP ${ }_{\mathrm{POx}}$ " and $" \mathrm{Ag}^{+} / \mathrm{Bi}^{3+}(\mathrm{XOR})-\mathrm{Au} \mathrm{NP}_{\mathrm{POx}}$ "- - designed by Lien and co-workers are promising components of the control system. These multiplex logic gates based on Au nanozymes opened a novel gate for the construction of Boolean algebra at nanoscale.

Later, Lien and his co-workers made use of the metal ion-based modulation of $\mathrm{Au}$ nanozymes into detecting applications. In this study, they induced $\mathrm{Pb}^{2+}, \mathrm{Hg}^{2+}$, $\mathrm{Pt}^{4+}$ and $\mathrm{Bi}^{3+}$ to fabricate the gates for the detection of ions- $\mathrm{Pt}^{4+} / \mathrm{Pb}^{2+}$ (AND) and $\mathrm{Bi}^{3+} / \mathrm{Hg}^{2+}$ (INHIBIT) via new combinations (Lien et al. 2014). Different from the former logic gates, $\mathrm{Pt}^{4+}-\mathrm{Au}$ and $\mathrm{Bi}^{3+}-\mathrm{Au}$ NPs are nanozymes with peroxidase-like activity, whereas the $\mathrm{Pb}^{2+}$ and $\mathrm{Hg}^{2+}$ ions are activators and inhibitors, respectively. These four metal ions acted as the input signals and the color reactions induced by the peroxidase-like activity were the output signals as well. In terms of the AND gate, only in the situation that the $\mathrm{Pb}^{2+}(1)$ and $\mathrm{Pt}^{4+}(1)$ were both deposited on the Au NPs logic gate simultaneously, the output would be $1(\mathrm{ON})$, otherwise it would be 0 (OFF). As for the INHIBIT gate, with the $\mathrm{Bi}^{3+}(1)$ and without the $\mathrm{Hg}^{2+}(0)$ on the $\mathrm{Au}$ $\mathrm{NPs}$, the gate transmitted $1(\mathrm{ON})$ as output signal. In addition, 0 (OFF) appeared in the case of both $\mathrm{Bi}^{3+}(1)$ and $\mathrm{Hg}^{2+}(1)$, with $\mathrm{Hg}^{2+}(1)$ and without $\mathrm{Bi}^{3+}(1)$, or neither of the ions. This Au NPs logic gate was designed to detect the metal ions in liquids, and even more, they combined these two gates to a rational gate networking, a logic circuit that promoted the visual detection for metal ions (Fig. 5). The $\mathrm{O}_{2}$ bubbles or reddish resorufin generated by enzyme reactions would show the $1(\mathrm{ON})$ output directly.

As we can see, various ions play an active role in modulating the activities of nanozymes. With easy modification and multiple enzyme-like activities, nanozymes may be an appropriate material for fabricating multiplex logic gates. Furthermore, it opens a new horizon of the way to build logic control network as well.

\section{The color change of cerium oxide nanozymes}

Alongside with the enzyme-like activities, some inorganic nanozymes also possess many special physicochemical properties related to the materials themselves. 
Fig. 4 The working models of logic gates based on five metals. A The $\mathrm{Hg}^{2+} / \mathrm{Bi}^{3+}(\mathrm{OR})-$ $\mathrm{Au} \mathrm{NP}$ CAT gate. $\mathrm{B}$ The $\mathrm{Pb}^{4+} /$ $\mathrm{Hg}^{2+}$ (AND)-Au NP $\mathrm{Ox}_{\text {gate. }}$ gat C The $\mathrm{Pb}^{2+} / \mathrm{Hg}^{2+}$ (INHIBIT)$\mathrm{Au} \mathrm{NP} \mathrm{POx}_{\mathrm{x}}$ gate. $\mathrm{D}$ The $\mathrm{Ag}^{+} /$ $\mathrm{Bi}^{3+}$ (XOR)-Au NP $\mathrm{POx}_{\text {gate. }}$ gat Reproduced with permission of Royal Society of Chemisty (Lien et al. 2013)
A

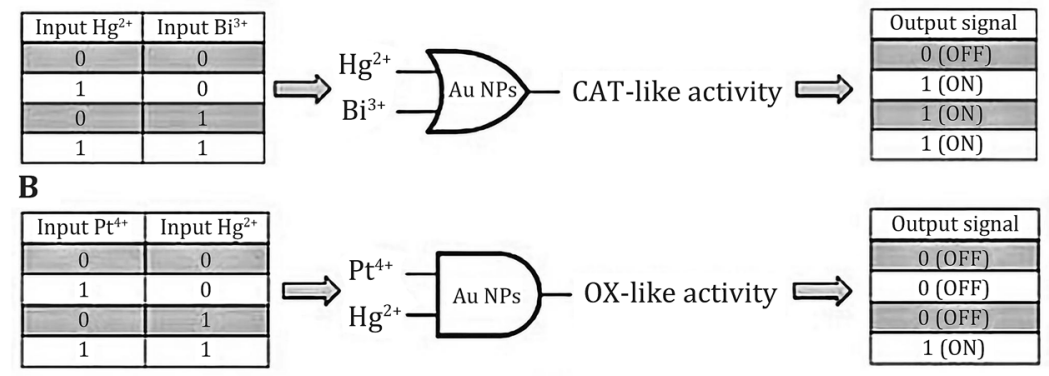

C

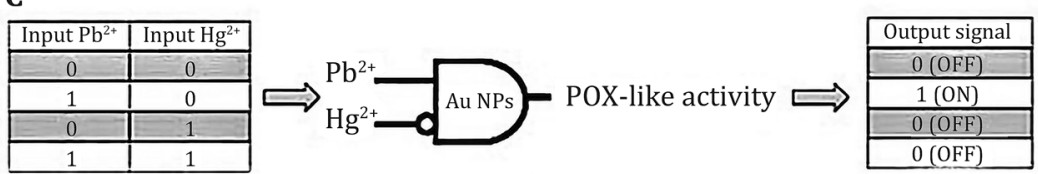

D

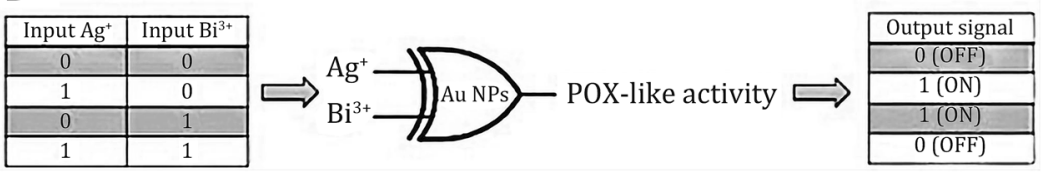

Fig. 5 The networking of metal ions detection. A The integrated circuit of logic gates. $\mathbf{B}$ The visible detecting outcome. a Absence of $\mathrm{Pb}^{2+}$ and $\mathrm{Hg}^{2+}$; $\mathbf{b}$ presence of $\mathrm{Pb}^{2+}$; c presence of $\mathrm{Hg}^{2+}$;

d presence of $\mathrm{Pb}^{2+}$ and $\mathrm{Hg}^{2+}$. C The table of inputs and outputs about $\mathbf{B}$ in the form of " 0 " and " 1 ". Reproduced with permission of American Chemical Society (Lien et al. 2014)
A

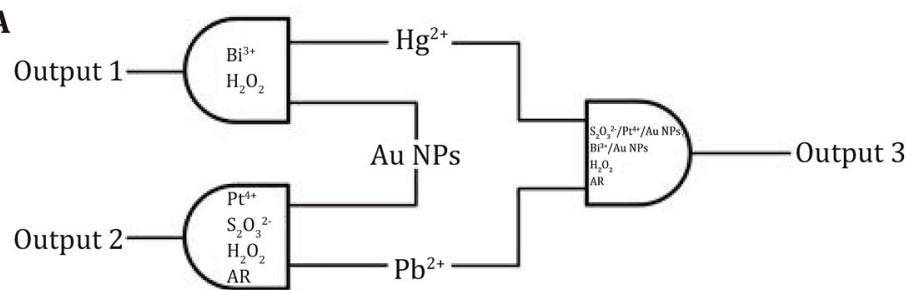

B

(a)

(b)

(c)

(d) $\mathrm{Au} \mathrm{NPs} / \mathrm{Bi}^{3+} / \mathrm{H}_{2} \mathrm{O}_{2}$

$\mathrm{Au} \mathrm{NPs} / \mathrm{Pt}^{4+} / \mathrm{S}_{2} \mathrm{O}_{3}{ }^{2-} / \mathrm{H}_{2} \mathrm{O}_{2} / \mathrm{AR}$

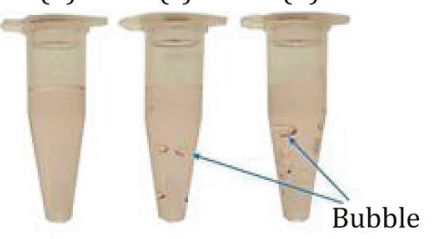

C

\begin{tabular}{ccccc}
\hline $\mathrm{Pb}^{2+}$ & $\mathrm{Hg}^{2+}$ & $\begin{array}{c}\text { Output } 1 \\
\mathrm{O}_{2} \text { bubble }\end{array}$ & $\begin{array}{c}\text { Output 2 } \\
\text { reddish resorutin }\end{array}$ & $\begin{array}{c}\text { Output 3 } \\
\mathrm{O}_{2} \text { bubble and } \\
\text { reddish resorutin }\end{array}$ \\
\hline 0 & 0 & 0 & 0 & 0 \\
\hline 1 & 0 & 0 & 1 & 0 \\
\hline 0 & 1 & 1 & 0 & 0 \\
\hline 1 & 1 & 1 & 1 & 1 \\
\hline
\end{tabular}


Among those nanozymes, cerium oxide nanozymes exhibit a unique characteristic-the color change of the nanozyme itself.

In 2007, Das et al. performed a series of experiments to investigate the auto-catalytic activity of ceria nanozymes and first observed the phenomenon of color change (Das et al. 2007). Later in 2008, Perez et al. further confirmed this result (Perez et al. 2008). At pH 7.4, when the hydrogen peroxide $\left(\mathrm{H}_{2} \mathrm{O}_{2}\right)$ was added, the solution went through a rapid color change from yellow to orange. After 10 days, the $\mathrm{H}_{2} \mathrm{O}_{2}$ decomposed and the color returned to yellow. The color change happens because of the reversible variation of $\mathrm{Ce}^{3+} / \mathrm{Ce}^{4+}$ ratio on the surface of the nanozymes. Singh et al. also synthesized cerium oxide nanozymes and observed the addition of $\mathrm{H}_{2} \mathrm{O}_{2}$ lead to the color change from colorless to yellow and the recovery after 15 days (Singh et al. 2011).

This reversibility of $\mathrm{Ce}^{3+}$ and $\mathrm{Ce}^{4+}$ on the surface of ceria nanozymes indicated its potential role as a perfect switch signal for the ON and OFF of the logic gate. Therefore, there has already been a study applying the ceria nanozymes to the Boolean algebra. Lin et al. utilized $\mathrm{CeO}_{2}$ nanozymes as signal transducer of the logic operation (Lin et al. 2012). This $\mathrm{CeO}_{2}$ nanozyme-based logic gate took $\mathrm{H}_{2} \mathrm{O}_{2}$ as $1(\mathrm{ON})$ then the signal would be transmitted by the color change from colorless to yellow. To reset the state of the gate back to OFF, it just needed a simple operation by heating the $\mathrm{H}_{2} \mathrm{O}_{2}$ to decomposition (Fig. 6A). As inputs, several enzymes in combination were constructed to AND, OR and INHIBIT gate including $\beta$-galactosidase ( $\beta$-Gal), glucose oxidase (GOx), xanthine oxidase (XO) and catalase (CAT) (Fig. 6B). For example, in the presence of lactose and oxygen, $\beta$-Gal and GOx would produce $\mathrm{H}_{2} \mathrm{O}_{2}$, leading to $\mathrm{ON}$ as output signal. Similar to AND gate, the OR and INHIBIT gate processed information in the environment with glucose and oxygen in the solution. Unlike the $\mathrm{Au}$ nanozyme-based gate mentioned before which simultaneously dealt with input signals together, this $\mathrm{CeO}_{2}$ nanozyme-based logic gate received the last information that has been through the cascading reaction. This design may offer a smarter logic algorithm for the control system. Even more, because of the relatively separated steps of the cascading reaction, the gates possess the ability to make an integration or a concatenation with each other (Fig. 6C). The computing ability may be regarded as a rudiment of the Boolean algebra calculation at nanoscale. Moreover, the complicated networks generated by these logic gates helped understand the complex biological systems. The $\mathrm{CeO}_{2}$ nanozyme-based logic gate may be utilized to mimic the natural biological pathways in cells.

\section{CuO:GNS nanozyme as the input signal}

Natural enzymes can be applied to the logic gate as input signals, and so can the nanozymes. Sharma and co-workers fabricated a simple AND logic gate to detect

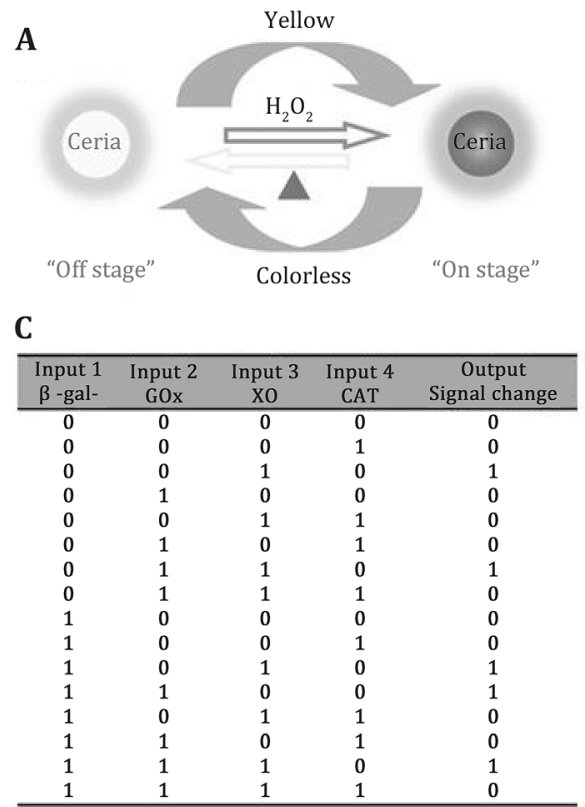

B

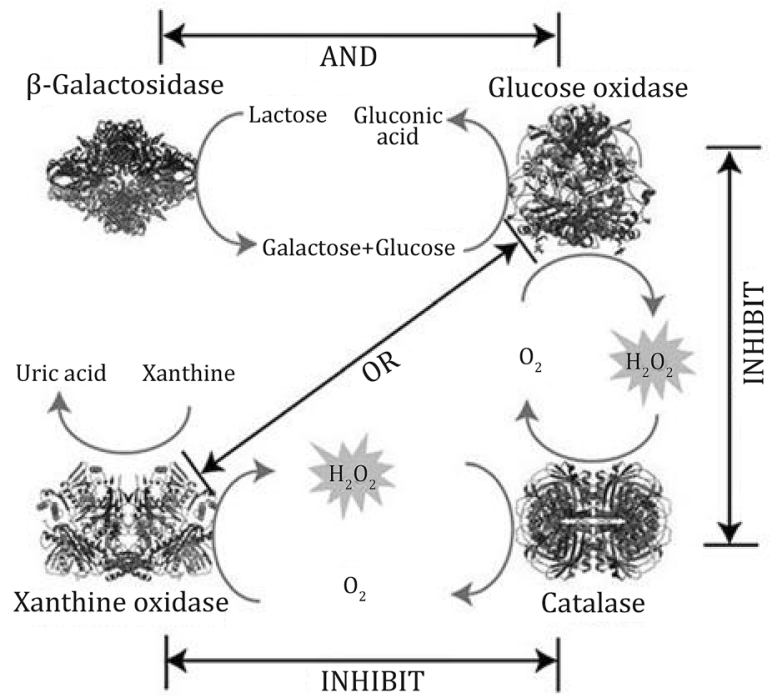

Fig. 6 The logic gates depended on the color change of $\mathrm{CeO}_{2}$ NPs. A The "ON" and "OFF" states and their corresponding color change. B The combination designs of AND, OR and INHIBIT gates and their corresponding catalytic reactions. $\mathbf{C}$ The integrated computing results of all inputs and outputs. Reproduced with permission of John Wiley and Sons (Lin et al. 2012) 
cholesterol by modifying the $\mathrm{CuO}$ nanozyme with graphene quantum dots (GQD) (Sharma and Mobin 2017). In the presence of cholesterol oxidase and chromogenic substrates (4-AAP and phenol), the modified CuO:GNS nanozyme with peroxidase-like activity and cholesterol were the input signal $(1,1)$, which led to the colorimetric reaction as output signal (ON). The cholesterol oxidase generated $\mathrm{H}_{2} \mathrm{O}_{2}$ in the oxidation reaction of cholesterols, then the CuO:GNS nanozyme catalyzed 4-AAP and phenol to red products, suggesting the generation of $\mathrm{H}_{2} \mathrm{O}_{2}$. Thus, the output signals could be detected by absorbance change. In addition, the modified CuO:GNS nanozyme has higher peroxidase-like activity than original $\mathrm{CuO}$ nanozymes. Therefore, the CuO:GNS nanozyme is also a good sensor for $\mathrm{H}_{2} \mathrm{O}_{2}$. Although simple structure limited its function, the CuO:GNS nanozyme-based logic gate is of cytocompatibility. GNS modification not only increases its catalytical activity, but also decreases the toxicity of $\mathrm{CuO}$ by generating lower levels of ROS. Hence, it is more proper to utilize in the living body, and it may be applied to a smart sensing system at nanoscale especially for medical use.

Nanozymes with enzyme-like activities and other special properties foster the building of logic gate for the control system. Moreover, with complex designs, nanozymes may even take an important step of the Boolean algebra computations at nanoscale, which is a significant contribution to the fabrication of nanorobots. Nanozymes are able to be applied to the utilization in vivo and in vitro, making them promising materials over a wild range of fields.

\section{Nanozyme-based driving system}

Taking notice of the characteristics in the fabrication of logic gates, we found that the catalyst of $\mathrm{H}_{2} \mathrm{O}_{2}$ has played an important role. $\mathrm{H}_{2} \mathrm{O}_{2}$ is a common fuel for the self-propelled nanomotors. Therefore, nanozymes are widely used in the field of driving system.

Nanorobots need a driving system to keep them moving as required and reach the targeting site. As we mentioned before, there are fuel-free and fueldependent nanomotors. In order to convert the fuels to power, the fuel-dependent nanomotors need catalysts, and enzymes may play an active role in generating power. For example, a mesoporous silica drug delivery nanoparticle system decorated with urease enzymes, not only enhanced the drug delivery, but also increased the drug release capability (Hortelão et al. 2017). It may be caused by the increased diffusion of fuel-powered nanomotor and the flow rate on the surface of drugloaded nanoparticle. Taking advantage of bioavailability and biocompatibility, it is ideal to convert the energy from the catalytic reaction to drive the nanorobot compared with other methods (Wang et al. 2019). Therefore, as a kind of promising enzyme-mimic materials, nanozymes are a better choice to power the driving system in nanorobots.

\section{Pt nanozyme-based linear motion}

Pt nanozyme, one of the metallic nanoparticles, possesses catalase/peroxidase-like activity, catalyzing $\mathrm{H}_{2} \mathrm{O}_{2}$ to generate $\mathrm{O}_{2}$ or oxidize other substrates. As we mentioned before, $\mathrm{H}_{2} \mathrm{O}_{2}$ is a common fuel for selfpropelled nanomotors. Hence, Pt nanozyme with catalase activity has been widely studied in fuel-dependent nanomotors, and some were engineered with other metals to enrich the functions or improve the abilities.

In 2004, F. Paxton et al. designed Pt/Au nanorods as nanomotors, which could be considered as the initial application of $\mathrm{Pt}$ nanozyme to power the motion of nanomachines (Paxton et al. 2004). In $\mathrm{H}_{2} \mathrm{O}_{2}$ solution, the $\mathrm{O}_{2}$ generated by $\mathrm{Pt}$ nanozyme formed an interfacial tension gradient to provide motion force (Fig. 7A), with the speed of approximately 2-10 body lengths per second. The movement of $\mathrm{Pt} / \mathrm{Au}$ nanorods was considered to be related to the electrokinetic mechanism of the redox reaction. In order to investigate the motion characteristics of the self-propelled micro/nanomotors, R. Howse and co-workers used an asymmetric spherical particle (so called Janus particles now) that only based on Pt nanozyme as the model for the nanomotor (Howse et al. 2007). They concluded that this nanomotor was powered by a different propulsion mechanism. Since then, Pt nanozyme has become a popular material for micro/nanomotors, such as Pt nanozyme-based particles (Choi et al. 2018), enhanced Pt nanorods (Kline et al. 2005), and Pt Janus particles (Gibbs and Zhao 2009). In the process of developing Pt nanozyme-based motors, scientists are working to increase the flow speed as well. Pumera's lab fabricated a micromotor for self-propelled drug delivery by combination of carbon nanotubes (CNTs) and Pt nanozyme (Khezri et al. 2019). The inner layer was constructed with Pt nanozyme, which catalyzed the $\mathrm{H}_{2} \mathrm{O}_{2}$ as fuels to power the micromotor. Then the reduced nanographene oxide deposited as outer layer via template-assisted electrodeposition (Fig. 7B). The catalase-like activity of inner Pt nanozyme catalyzed the fuel $\mathrm{H}_{2} \mathrm{O}_{2}$ to oxygen and water, so that the generated $\mathrm{O}_{2}$ could push the micromachine into movement by bubble ejection. The average velocity was $588 \mu \mathrm{m} / \mathrm{s}$ in $3 \% \mathrm{H}_{2} \mathrm{O}_{2}$ of the tubular micromachines, and the maximum reached $700 \mu \mathrm{m} / \mathrm{s}$, which is noticeably faster than other Pt nanozyme-based motors. After the loading of doxorubicin (DOX), this micromotor could 
A

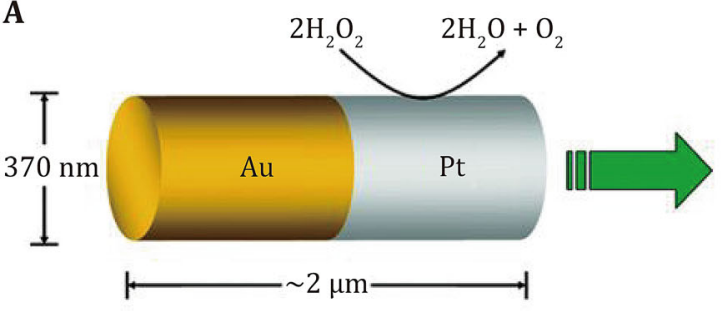

B

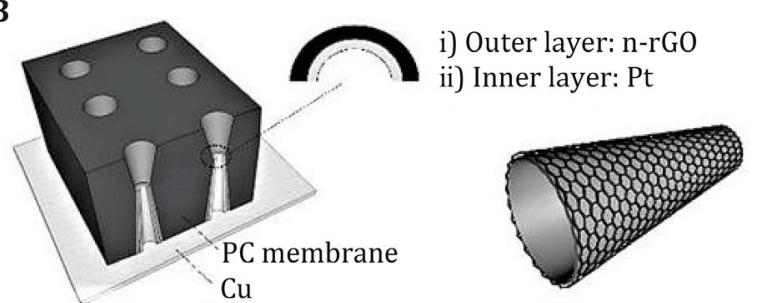

Fig. 7 Pt nanozyme-based nanomotors. A The design of the first Pt nanozyme-based nanomotor. Reproduced with permission of American Chemical Society (Paxton et al. 2004). B The structure of Pt nanozyme-based carbon nanotubes. Reproduced with permission of John Wiley and Sons (Khezri et al. 2019)

deliver drugs to cancer cells. Considering that cancer sites are usually accumulated with $\mathrm{H}_{2} \mathrm{O}_{2}$, Pt-based nanomotors may make a great contribution to cancer therapy.

\section{Other materials and motion patterns}

Pt nanozyme is the most common nanomaterial used to power the micro/nanomotors, probably because of its highly efficient catalytic property in the propelling utilization. However, there are other materials for building motors, which also possess catalytical activities or combine with Pt nanozyme to help the construction. Besides, based on multiple materials, these motors may act in other ways like rotation.

Fournier-Bidoz et al. designed a nanorod by means of decomposing $\mathrm{H}_{2} \mathrm{O}_{2}$ on the surface of nickel nanorods to generate $\mathrm{O}_{2}$ bubbles (Fournier-Bidoz et al. 2005). The two segments of these nanorods were made of gold and nickel respectively and the gold end was anchored to the silicon substrate, so the whole nanorods made a rotational movement at a speed of $1.5 \pm 0.2 \mathrm{rad} / \mathrm{s}$. He et al. further designed a boomerang-like structure for the rotation (He et al. 2007). Si made up of L-shape backbones with a longer arm and a shorter arm, and the Pt nanozymes deposited on the longer arm asymmetrically (only on one side of the nanorod) which allowed the L-shape nanorod to do rotary motion. (Fig. 8A, B). Then they also deposited Ag to the side of nanorods, and found they moved faster than Pt nanozyme (Fig. 8C). $\mathrm{H}_{2} \mathrm{O}_{2}$ was catalyzed on the surface of $\mathrm{Ag}$ nanozyme and generated $\mathrm{O}_{2}$ bubbles to propel the rotation, similar to the Pt nanozyme. Furthermore, two Ag-based nanorods were even linked to form a nanospring resulting in a helically rotating movement (Fig. 8D). However, the biotoxicity of Ag nanozymes largely restricts its applications. Vicario designed a rarely used material, manganese, to catalyze $\mathrm{H}_{2} \mathrm{O}_{2}$ fuels, the main body and $\mathrm{Mn}$ based power engine of which were independent parts and linked by a tether.(Vicario et al. 2005). Functioning as an engine, molecular $\mathrm{Mn}\left(\mathrm{ClO}_{4}\right)_{2}$ possessed the catalytic activity to generate $\mathrm{O}_{2}$ from $\mathrm{H}_{2} \mathrm{O}_{2}$. Interestingly, linear and rotary movements were both observed in these experiments (Fig. 8E, F).

\section{Nanozyme guidance in motion control}

Based on above analysis, we have enabled the autonomous movement of nanomotors with fuels, and there comes a further problem: how can we control the movement? Nanomotors without control will just be propelled to do random motion, so they are not useful for practical application. Thereby, we need to present nanorobots with the ability of reaching the expected place through directed trajectory. Taking the nanomotor as the engine of a car, we need steering wheels, a braking and an accelerator to manage the speed and orientation, so that nanomotors are able to get to the targeting position. Hence, the control of motion will help the nanomotor get to the appointed place with minimum fuels and fast speed, and without unwanted movement. Magnetic or chemical approaches play a part in the external or internal control, which boost the utilization of nanozymes. Consequently, nanozymes with magnetism and other abilities are favorable in motion control by responding to the environment or signals.

On the contrary of nanomotors, some nanorobots are not endowed with driving systems, but they also need to reach the targeting place. When those nanoparticles are injected into living bodies, their movement usually depends on the blood flow and they accumulate in the tumor site through the enhanced permeability and retention (EPR) effect. Therefore, some nanoparticles are modified with antibodies to target the cancer cells. Nanozymes are easy to be modified with cancer-targeting antibodies, and for this reason, these nanozymes are suitable materials to help guide the nanorobots to the targeted places. 
A

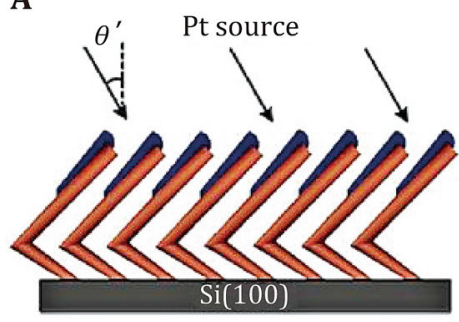

C

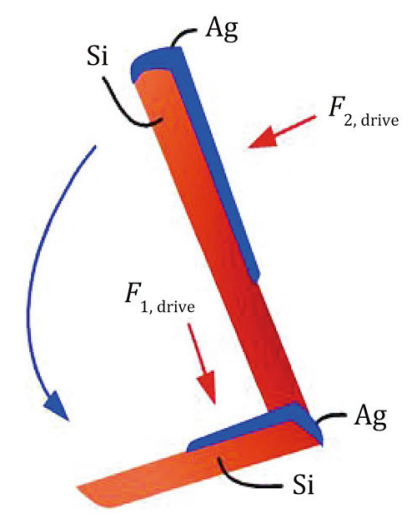

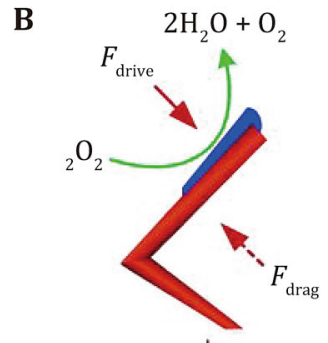

D

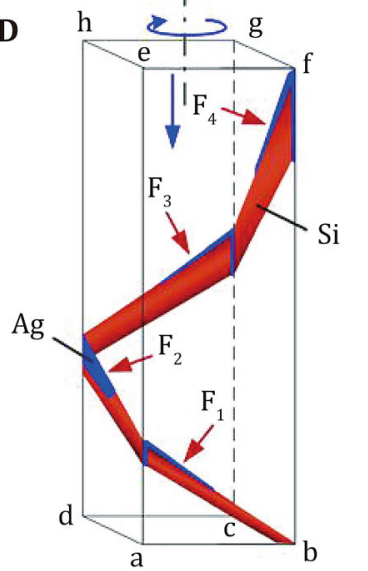

$\mathbf{E}$

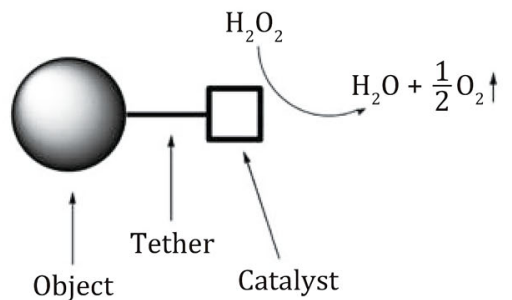

F

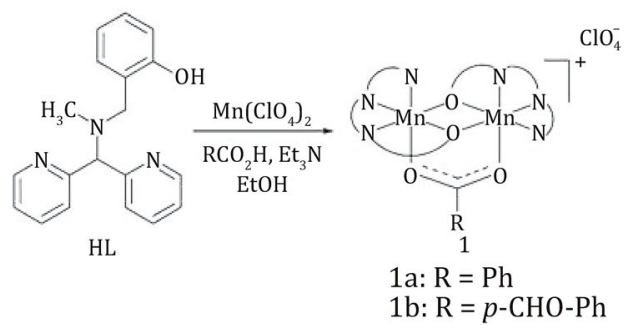

Fig. 8 The fabrication (A), propelling mechanism and force (B) of the Pt-nanozyme based L-shape nanomotors. The fabrication (C), nanospring and helically rotating movement (D) of the Ag-based L-shape nanomotors. Reproduced with permission of American Chemical Society (He et al. 2007). The structure (E) and catalytic molecule (F) of the Mn-based nanomotor. Reproduced with permission of Royal Society of Chemistry (Vicario et al. 2005)

\section{Magnetic guidance}

Magnetic field is a common choice for wireless control of many devices at macroscale, microscale or even nanoscale, and the iron oxide nanozyme is a general material for magnetic guidance. Some microorganisms possess nanometer-sized magnetosomes inside the cells, so they take advantage of the magnetic field to help the movement. This property inspired some scientists to design a kind of bionic micro/nanorobots to mimic the movement of magnetotactic bacteria. For example, Kim and co-workers employed iron-oxide nanozymes (magnetite) to create an artificial Tetrahymena pyriformis (Kim et al. 2010) (Fig. 9A-C). The manmade cells could move much more quickly than many microorganisms, with an average speed at $786.7 \mu \mathrm{m} / \mathrm{s}$.

In the former part we have described the fueldependent motors powered by metals, which have a strong engine. However, nano/micromotors need a steering wheel to guide their direction, and magnetic metals have shown their potential. $\mathrm{Fe}_{3} \mathrm{O}_{4}$ nanozyme is a most common nanozyme with magnetism, therefore it has been used in many nanomotors for the magnetic guidance. Villa engineered a multifunctional Janus particle decorated with Pt nanozyme as a fuel-powering engine, which could be self-propelled in $\mathrm{H}_{2} \mathrm{O}_{2}$ solution. (Villa et al. 2018). Coated with a porous polymeric shell, the iron oxide nanozymes were encompassed in the inner part of the Janus particle, endowing it with magnetic features to manipulate the motion of microrobots in external magnetic field. In addition, the outside surface of this microparticle was decorated with tosyl groups to capture the cancer cells or deliver DOX. When controlled by a magnetic field, the particles would form a chain-like spherical structure, and the cells released by trypsin would bind with the decorated microparticles (Fig. 9D). By adjusting the magnetic guidance, the Janus particles could alter their direction and took the cells into movement. The cells attached to the surface of the particles firmly, without breakage of bonds while moving with the chain-like particles. In addition, six to seven Janus particles generated adequate force to drag one or even more cancer cells. On the other hand, loading with DOX, the self-powered microrobots were guided to the cancer site by magnetic field, then released drugs to cancer cells. This multifunctional designment will provide a great inspiration for nanorobot fabrication. 

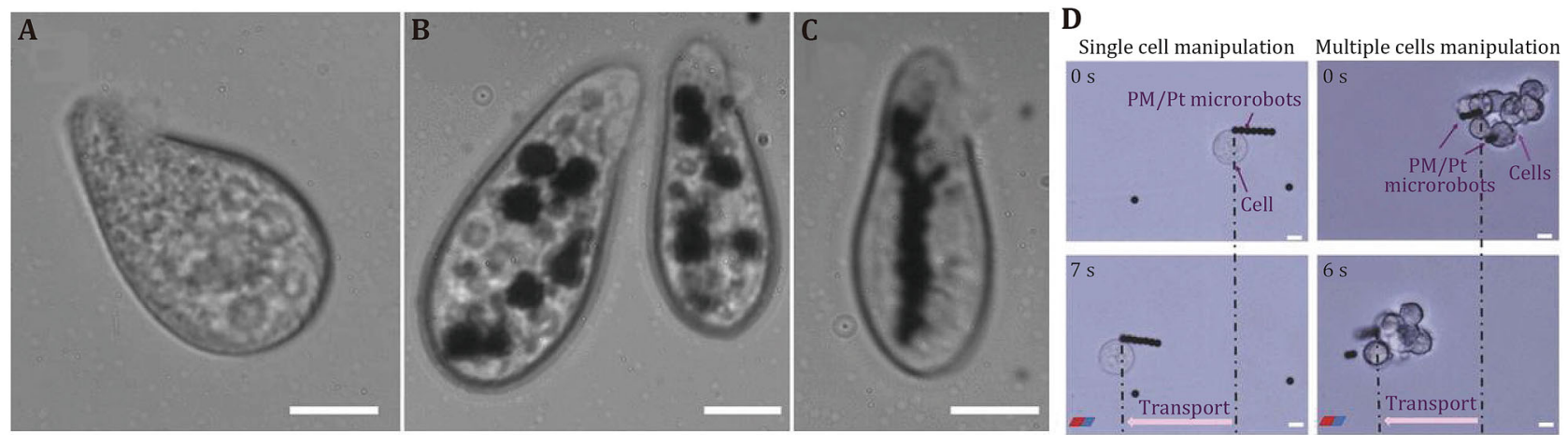

Fig. 9 Normal status without artificial modification (A), internalization of iron oxide nanozymes into cells (B), magnetization of internalized iron oxide nanozymes (C) of T. pyriformis. The scale bars are $10 \mu \mathrm{m}$. Reproduced with permission of AIP Publishing (Kim et al. 2010). D The dragging of cancer cells by chain-like particles. Reproduced with permission of John Wiley and Sons (Villa et al. 2018)

Furthermore, magnetic guidance is able to combine with other methods to steer the motion. The gold/iron oxide nanozyme-based nanorods made by Zhou were manipulated by the visible light and magnetic field (Zhou et al. 2017). These nanorods with properties of semiconductors transferred light energy to chemical energy by taking advantage of the photoelectric effect. Therefore, the visible light could control the power of the nanorods in $\mathrm{H}_{2} \mathrm{O}_{2}$ solution-the switch of on and off, and the adjustment of speed. With the magnetic features of iron oxide nanozyme, the whole nanorods are able to be directed by magnetic field.

\section{Environment-responsive chemical control}

Apart from the wireless control from external devices, the motion of nanorobots also depends on the response to environment. Some nanomachines have the sensing system, so they attain information from surrounding environment through the sensing system, then send instructions to the driving system. While the others can be instructed by the environment directly. The concentration of fuels affects the speed control. Unlike the machines at macroscale which contain fuels inside, micro/nanomachines usually utilize fuels in the environment. Hence, by altering the concentration of fuels in the aqueous solution, the velocity will be modulated accordingly. In a similar way, components in the aqueous solution have an impact on the speed as well. Laocharoensuk and his partners made an effort to modulate self-propelled nanomotors by changing components in solutions. They fabricated the nanomotor by adding carbon nanotubes (CNT) into the $\mathrm{Au} / \mathrm{Pt}$ nanozyme-based nanotubes and tested the speed in concentration gradient of $\mathrm{H}_{2} \mathrm{O}_{2}$ solution (Laocharoensuk et al. 2008). This nanomotor was as same as others, speeding up as the concentration increased. Notably, hydrazine induced an acceleration, demonstrating that certain components in the environment accelerated the self-powered nanomotor (Fig. 10). In addition, silver ions were reported to accelerate an Au-Pt nanozyme-based nanowire powered by $\mathrm{H}_{2} \mathrm{O}_{2}$ solution (Kagan et al. 2009). Among 11 metal cations in the solution, it was found that $\mathrm{Ag}^{+}$dramatically increased the speed (Fig. 11). This might result from the underpotential deposition of $\mathrm{Ag}$ metals on the surface of the Au-Pt nanozyme-based nanomotor, which affected its catalytic efficiency similar to the control of nanozyme-based logic gates discussed before. Speed changes are able to be used for detecting silver ions. By virtue of altering the components in the complex solutions, it is convenient to control the motion of nanomotors via a novel method.

Through altering components in the environment, this way of motion control seems a little rough at nanoscale, but it still provides a reference. Furthermore, it may be a better idea to adjust the concentration in the environment for motion control, which is similar to the chemotaxis of microorganisms. Living cells are able to orientate the movement of themselves by following the lead of concentration gradients. This phenomenon not only happens within microorganisms but also appears in the cells of multicellular organisms (Van Haastert and Devreotes 2004). Once again, inspired by the intelligence of nature, scientists are seeking for the applications of chemotaxis in the guidance of artificial micro/nano devices. R. Howse and his partners studied the motion of $\mathrm{Pt}$ nanozyme-based nanomotors and observed the speed-concentration relationship and the feature of directions (Howse et al. 2007). They found that Pt nanozyme-based nanomotors exhibited a directed motion in a short time, although this phenomenon disappeared at longer times. And the higher the concentration of $\mathrm{H}_{2} \mathrm{O}_{2}$ solution was, the longer time of the directed motion lasted. This might be 

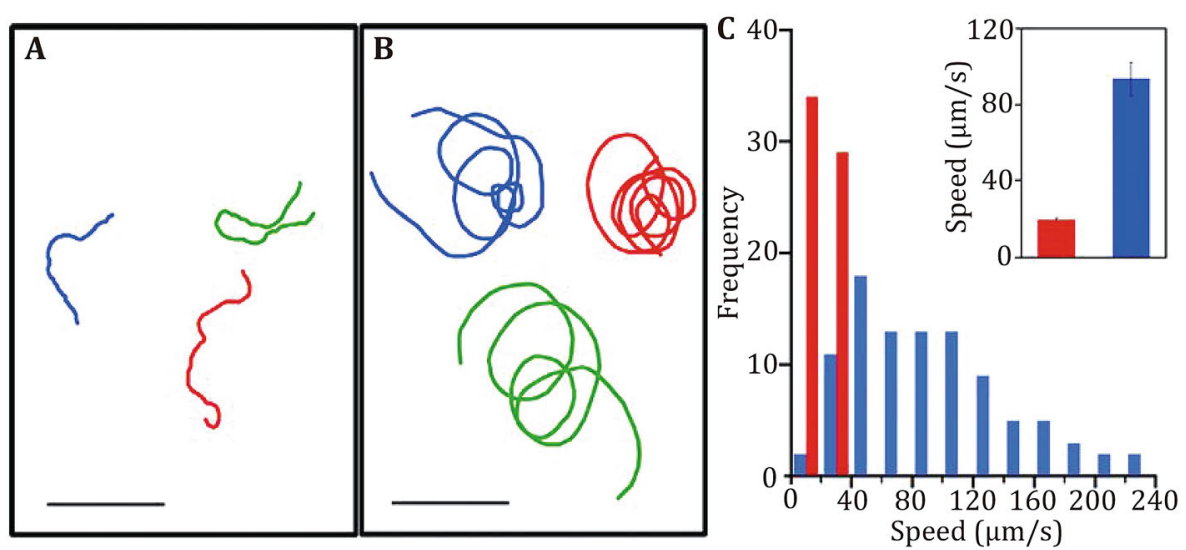

Fig. 10 Influences of hydrazine on the CNT nanomotors. Tracking lines about the movement of nanomotors in the solution without (A) and with hydrazine (B). Scale bar is $50 \mu \mathrm{m}$. C Illustrating of the speed distribution without (red) and with (blue) hydrazine. Reproduced with permission of American Chemical Society (Laocharoensuk et al. 2008)

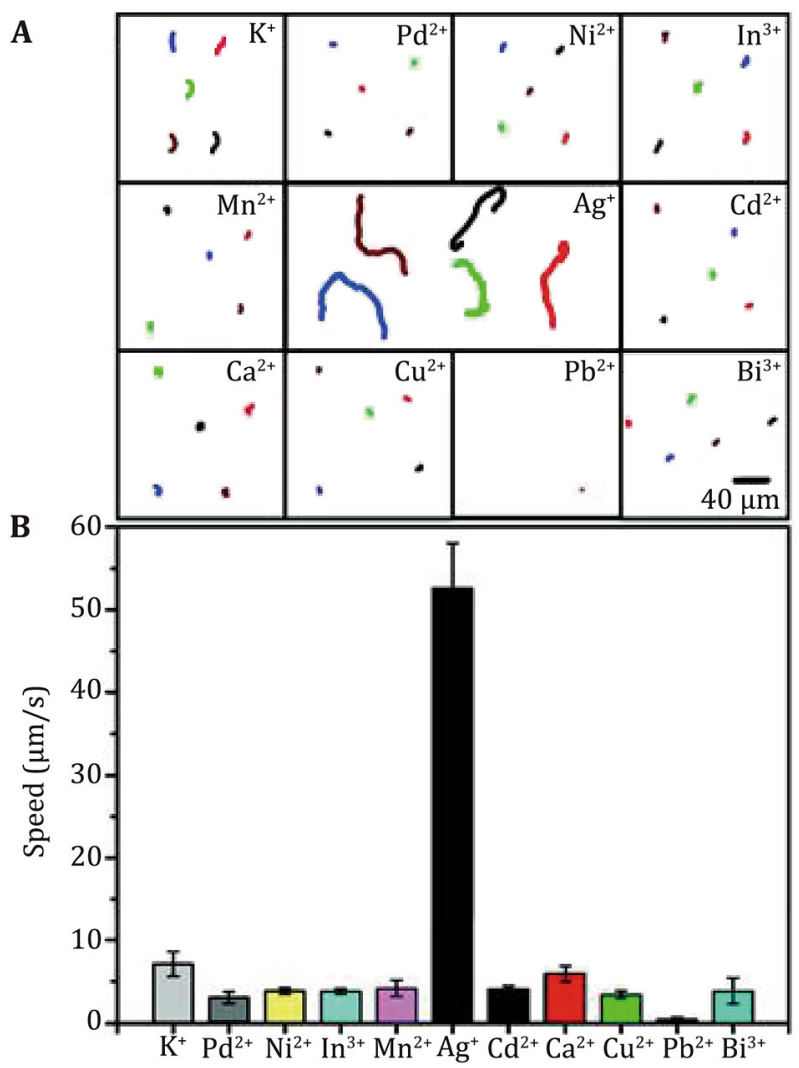

Fig. 11 Motion of nanomotors in solutions containing 11 different metal ions respectively. Tracking lines about the movement of nanomotors (A) and illustrating of the speed (B) in solutions with 11 different metal ions respectively. Reproduced with permission of American Chemical Society (Kagan et al. 2009) the first glance at the chemotaxis of artificial micro/ nano devices outside the biological system. Ayusman Sen's team built the first Pt nanozyme-based nanomotor in 2004 (Paxton et al. 2004), and later in 2007, they continued to study the chemotaxis of their nanomotor (Hong et al. 2007) (Fig. 12). Experiments demonstrated that many of the nanomotors accumulated at the site with high concentration. In addition, in the model of concentration gradients, the number of nanomotors gathered in $10 \% \mathrm{H}_{2} \mathrm{O}_{2}$ solution was larger than that of $1 \%$. These results presented the chemotaxis of nanomotors. Moreover, two types of nanomotors were demonstrated to follow the rule of chemotaxis although powered by different propulsion mechanisms (Baraban et al. 2013). In this experiment, the Janus particles were more sensitive than the tubular microjets when put in the concentration gradient of $\mathrm{H}_{2} \mathrm{O}_{2}$ solution, which was related to their shape. Therefore, the characteristics of chemotaxis are relevant to the size and shape as well. Considering that the cancer site possesses higher concentration of $\mathrm{H}_{2} \mathrm{O}_{2}$, chemotaxis may be a promising guidance for the micro/nanomotors applied in the living body.

In addition, $\mathrm{pH}$ is also a good tool for steering the orientation, such as the novel submarine-like nanomotor designed by Liang's lab (Guo et al. 2019). This nanomotor seemed like a submarine minimized to nanoscale, for the mechanisms modulating it was just the same as the real submarine-related density functional theory (DFT). Metal-organic framework (MOF) nanozyme acted as the propelling engine, which mimicked the catalase (CAT) activity. And MOF nanozymes were decorated with special $\mathrm{pH}$-responsive materials as the sensing system (Fig. 13A). Polymer 
A

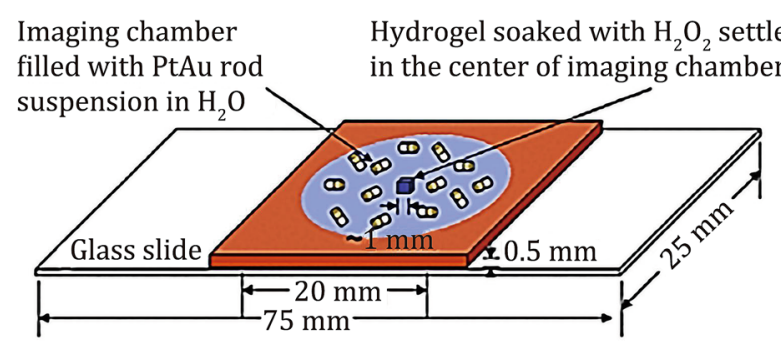

B

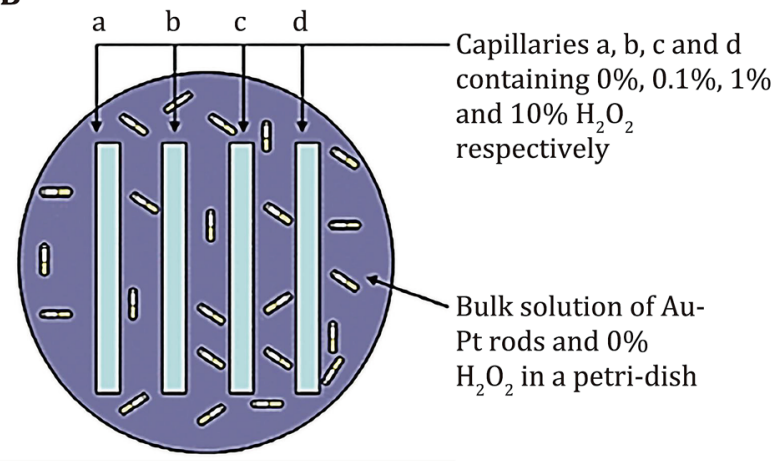

Fig. 12 Solution fields for the chemotaxis detection of nanomotors. A A gel contained $30 \% \mathrm{H}_{2} \mathrm{O}_{2}$ in the center of the water solution with nanomotors. B Four gels with $\mathrm{H}_{2} \mathrm{O}_{2}$ gradient in the water solution with nanomotors. Reproduced with permission of American Physical Society (Hong et al. 2007)

poly(2-diisopropylamino) ethyl methacrylate (PDPA) could shift the phase of hydrophilicity or hydrophobicity, helping the nanomotor respond to the environment change. The MOF nanozymes catalyzed the generation of $\mathrm{O}_{2}$ bubbles in $\mathrm{H}_{2} \mathrm{O}_{2}$ solution, and when $\mathrm{pH}$ was above 6.4 , the bubbles would attach to hydrophobic PDPA, and then accumulated buoyancy force was strong enough to lead the nanomotor to flow up. On the contrary, if the $\mathrm{pH}$ was below 6.4 , bubbles would dissociate with hydrophilic PDPA, leading to the downward movement. The movement of this nanomotor could be achieved at centimeter scale, and even could be visualized via bare eyes (Fig. 13B). This original idea opens a new gate for the strategies of motion control.

Chemical motion control depending on the environment is an available way to fabricate the nanomotors. Because of the complexity and easy-modulation of the aqueous environment, it is always a popular strategy.

\section{Modified nanozymes for targeting}

For the nanodevices used in living body, there is also a convenient way to help guide them to accumulate at the targeting positions or to interact with objects: decorating the target molecules on the surface, such as ligands for receptors on the cells. It seems like a simple sensing system that senses objects in specific places, and then the decorated nanodevices will attach to the objects and further transmit information to the whole nanorobots. These nanodevices only function when the distance is close enough, so most of the nanodevices without driving system depend on the blood flow inside the body. The artificial engineered platelet we discussed before is a typical example ( $\mathrm{Hu}$ et al. 2016).

Recently, Ding et al. built exosome-like nanozyme vesicles for clinical cancer diagnosis in vivo (Ding et al. 2018). The horseradish peroxidase-like graphene quantum dot nanozyme (GQDzyme) together with the 2,2'-azino-bis (3-ethylbenzothiazoline-6-sulfonic acid) (ABTS) was encompassed in the natural erythrocyte membranes (RM) to form the vesicle. Folate acid (FA) was conjugated on the outside surface of the particle, so that the nanoparticles were able to target nasopharyngeal carcinoma (NPC) cells through the interaction with the overexpressed folate receptors (FR) on these cancer cells. With horseradish peroxidase (HRP)-like activity and the high level of $\mathrm{H}_{2} \mathrm{O}_{2}$ in the cancer tissues, GQDzyme inside the nanoparticles oxidized ABTS, and the near-infrared (NIR) absorbance of oxidized ABTS would be a suitable contrast agent for photoacoustic imaging (PAI).

Decorating target molecules are indeed a good strategy, and a nanocage exhibiting intrinsic targeting ability is a favorable choice for nanoparticle fabrication. Human H-ferritin (HFn) binds to transferrin receptor 1 (TfR1) on many cells without extra modification, especially the ones overexpressed in tumor cells (Fan et al. 2012). With a cage-like structure, ferritin is a perfect nanocarrier itself. Moreover, recently it has been reported that HFn has the ability to cross through the blood brain barrier (BBB) (Fan et al. 2018a). Because TfR1 is also overexpressed on the surface of the BBB endothelial cells (ECs), Dox-loaded HFn was found to deliver drugs to glioma cells by overcoming the $\mathrm{BBB}$ (Fig. 14A). In addition, ferritin can be easily modified via a genetic engineering approach (Jiang et al. 2019a). By linking the hepatocellular carcinoma (HCC)-targeting peptide SP94 to the subunit of ferritin, the specific binding ability for particular tumor cells largely increased. Meanwhile, the modified ferritin could still self-assemble and exhibited SP94 on the exterior surface. As a proper nanocage for carrying materials, the 
A

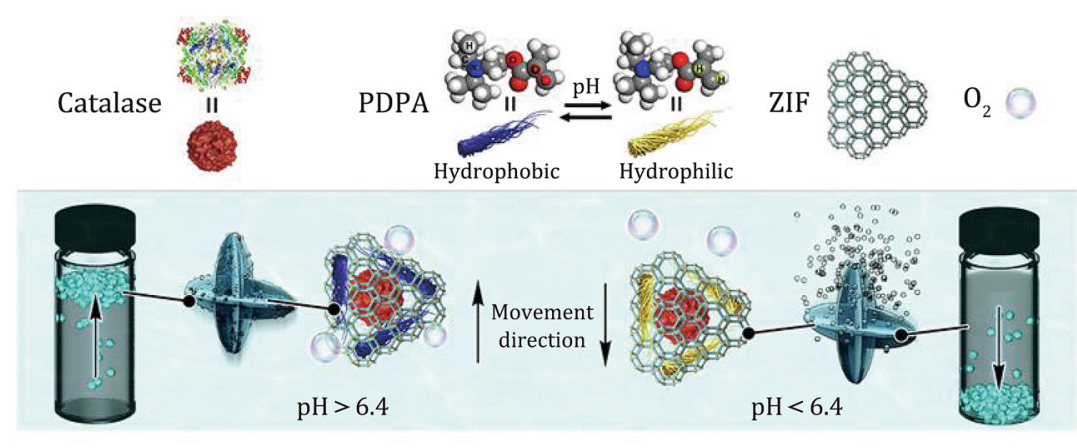

B

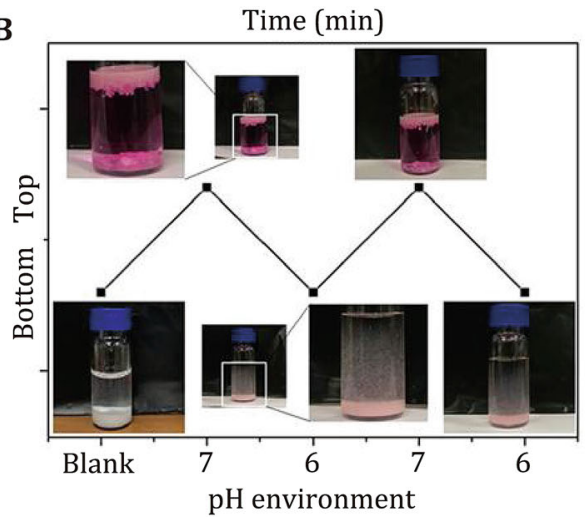

Fig. 13 The pH-responsive submarine-like MOF micromachines. A The fabricating materials and illustrating of pH-responding movement. B The visible movement of pH modulation. Reproduced with permission of Elsevier (Guo et al. 2019)

A

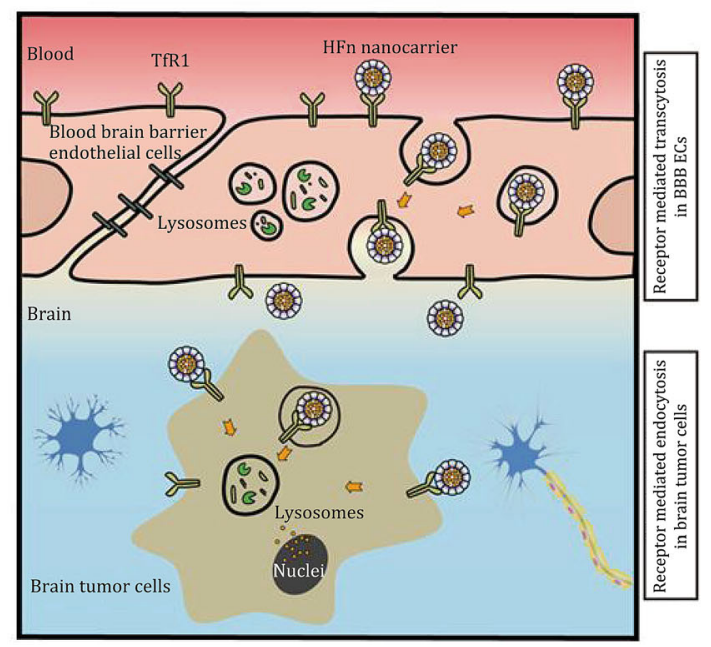

B

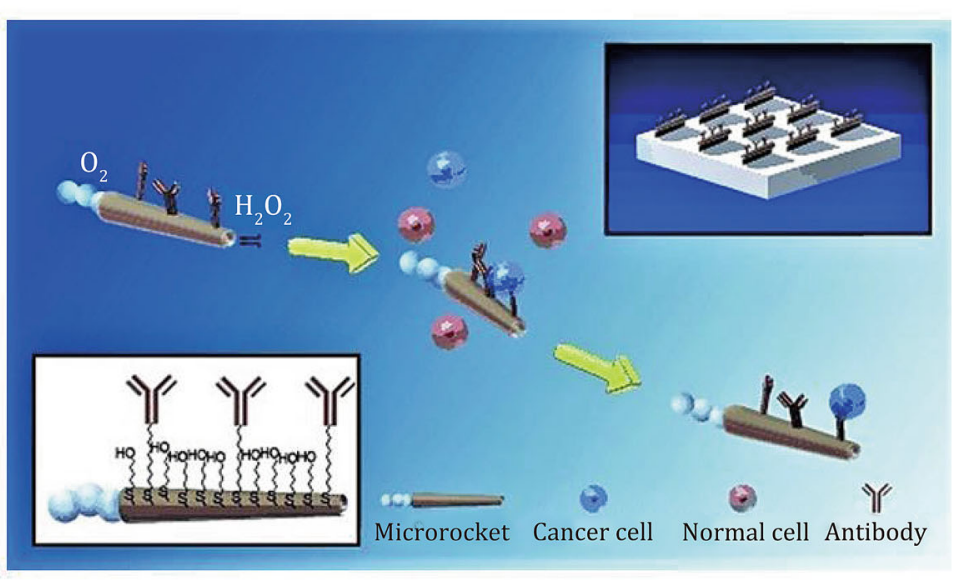

Fig. 14 A HFn crossed the BBB through TfR1. Reproduced with permission of American Chemical Society (Fan et al. 2018a). B The antibody-decorated microrocket captured CTCs. Reproduced with permission of John Wiley and Sons (Balasubramanian et al. 2011)

modified ferritin contained horseradish peroxidase-like $\mathrm{Co}_{3} \mathrm{O}_{4}$ nanozyme was demonstrated to stain the clinical HCC tissues in the presence of $\mathrm{H}_{2} \mathrm{O}_{2}$ and DAB.

It seems a little crude to apply this targeting strategy to the motion control for unpowered nanoparticles, but it increases the precision in powered micro/nanomachines. A decorated microrocket was reported to catch the circulating tumor cells (CTCs) in fluids because of the target ligands on the surface (Balasubramanian et al. 2011). The engine of this microrocket was built by $\mathrm{Pt}$ nanozymes in the inner layer and the middle layer is constructed by ferromagnetic (Fe) nanozymes to guide the engine with external magnetic fields. Finally, the monoclonal antibody (mAb) was conjugated to the outer gold surface. The anti-carcinoembryonic antigen (anti-CEA) has been demonstrated to be overexpressed in some common cancer cells, so the anti-CEA mAb could guide the micromachine to catch CTCs, and then took them to directed place (Fig. 14B). This microrocket might contribute to the early diagnosis of cancers including colorectal, gastric, and pancreatic carcinoma.

Nanozymes with magnetism and chemical-responsive properties are favorable for the motion control of micro/nanomachines. In addition, their easymodification also provides a convenience for the 
guidance of targeting molecules, in which case, ferritin is especially noticeable.

\section{Nanozyme for the function system}

Nanorobots are widely studied in medical applications. Many smart nanodevices have been designed to realize more complex functions than before. In addition, smart nanodevices perform tasks efficiently in vitro. However, they usually lack the driving system and get to the target site by EPR effect and contact with the objectives with or without modified targeting molecules.

To enhance the practical utilization of nanorobot, scientists selected nanodevices with smart functions and endowed them with systems like driving system and sensing system to build useful nanorobots. In many cases, nanozymes play an important role in the endeavor of providing functions for the nanodevices. Here, we list some designs of functions which have been achieved by nanozymes such as the detection and therapy of tumor and antimicrobial applications, etc. These examples may offer novel ideas to help design the function system.

\section{Tumor theranostics}

Tumor theranostics remains a troublesome problem for many years and scientists have taken a number of endeavors to investigate it. After the discovery of nanozyme in 2007, scientists found that nanozyme may be a proper choice for tumor theranostics and some of them have already been utilized for tumor detection and therapy.

nanozymes have shown potential in tumor therapy. Based on the ROS damage of cells, Qu's lab designed a multi-nanozyme nanoflowers to kill tumor cells (Wang et al. 2018) (Fig. 15A, B). They combined two types of nanozymes, which were the PtCo nanozymes with intrinsic oxidase-like activity and the $\mathrm{MnO}_{2}$ nanozymes mimicking catalase (CAT). These materials were able to self-assemble into a whole structure step by step. Especially, the PtCo nanozymes could direct the growth of $\mathrm{MnO}_{2}$ nanozymes. The $\mathrm{MnO}_{2}$ nanozymes with the intrinsic catalase-like activity converted high level of $\mathrm{H}_{2} \mathrm{O}_{2}$ in tumor cells to $\mathrm{O}_{2}$, then the PtCo nanozymes catalyzed the cascades of oxidation reaction via oxidase-like activity. Therefore, the generated ROS damaged a large part of components in the cancer cells resulting in apoptosis and necrosis. This function system did not need an external supply of $\mathrm{O}_{2}$ to generate $\mathrm{ROS}$ and this $\mathrm{MnO}_{2} @ \mathrm{PtCo}$ nanoflower exhibited high catalytic activity in the acid solution (Fig. 15C). This characteristic means that the
$\mathrm{MnO}_{2} @ \mathrm{PtCo}$ nanozyme is very appropriate for destructing tumor cells, which contain high level of $\mathrm{H}_{2} \mathrm{O}_{2}$ and are of acid $\mathrm{pH}$. Moreover, the results showed little cytotoxicity towards normal cells, thus it is an ideal choice for in vivo therapy.

\section{Antimicrobial functions}

Besides the applications for tumor theranostics, nanozyme-based particles are developing in the area of antimicrobes as well.

Nanozymes such as cerium oxide $\left(\mathrm{CeO}_{2}\right)$ and iron oxide $\left(\mathrm{Fe}_{2} \mathrm{O}_{3}\right)$ nanozymes (Masadeh et al. 2015), $\mathrm{Fe}_{3} \mathrm{O}_{4}$ nanozymes (Gao et al. 2014) and Au nanozymes (Ayaz Ahmed et al. 2014; Mohamed et al. 2017; Wang et al. 2017b; Yang et al. 2017) are employed for the obliteration of unwanted microbes. These nanozymes are capable of generating ROS to destruct biofilms of bacteria. Among the antimicrobic applications, there is a special scheme which functions in the seawater environment (Natalio et al. 2012) (Fig. 16). Vanadium pentoxide nanozyme with peroxidase-like activity could produce hypobromous acid and singlet molecular oxygen under seawater conditions, against the marine bacteria, which promote the prevention of marine biofouling on the shells of ships. Besides the stability of such nanozymes, it has been demonstrated that the vanadium pentoxide nanozymes are not harmful to marine biota.

\section{Other functions}

As we can see, nanozymes are more easily combined to work as multi-functional catalysts compared with natural enzymes. Particularly, the metal-organic framework (MOF) possesses large surface and highly ordered porous structure. Zhang's lab took advantage of this characteristic and built a function system to mimic natural cascade reactions (Huang et al. 2017). The MOF nanozyme possessed intrinsic peroxidase-like activity, then the $\mathrm{Au}$ nanozyme with glucose oxidase (GOx)-like activity grew on the surface of MOF nanosheets. Therefore, the two catalytical activities were combined together, and in the presence of $\mathrm{O}_{2}$, the multifunctional nanozyme oxidized glucose to gluconic acid and $\mathrm{H}_{2} \mathrm{O}_{2}$ and the latter would continue reacting with TMB because of the peroxidase-like activity. The multifunctional nanozymes were used to detect glucose and helped investigate the mimicking of natural enzymic cascade reactions. Moreover, as a biocompatible material, Au-modified MOF is suitable for the in vivo therapy and has been studied in cancer treatments. In this scheme, decorated with Au nanozymes, the newly synthesized MOF nanozyme possessed GOx-like and 

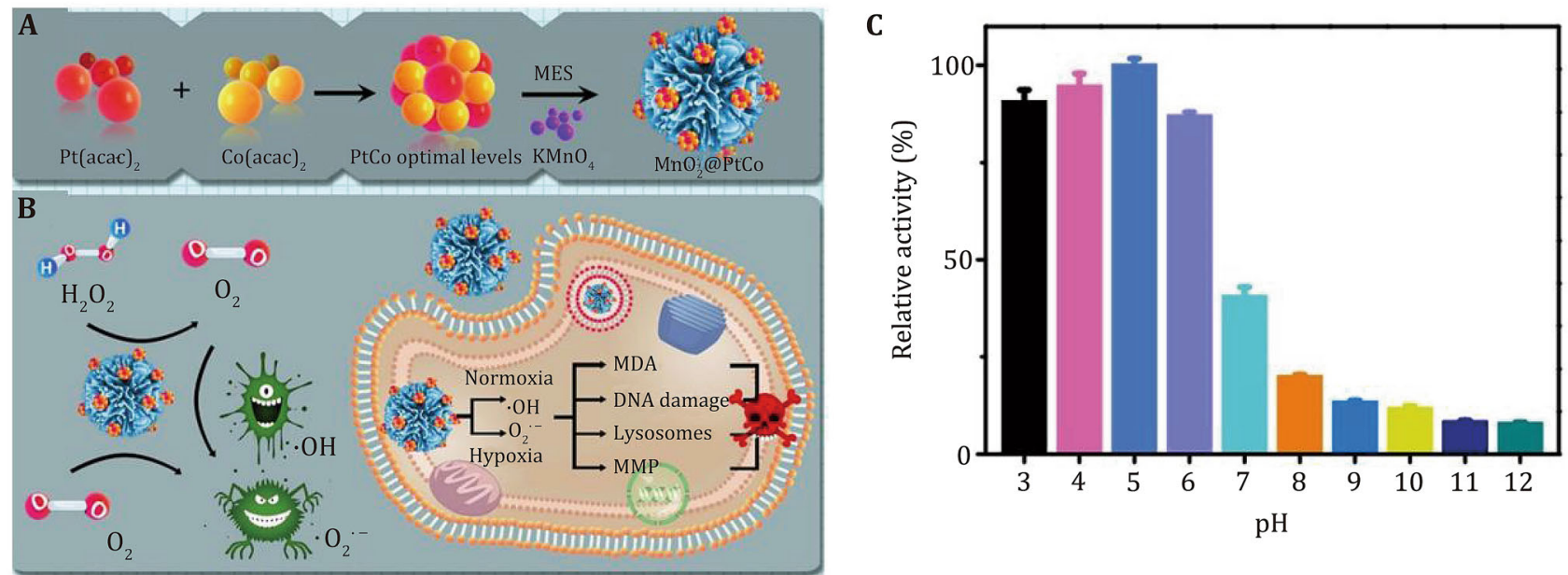

Fig. 15 Multi-nanozyme nanoflowers for killing tumor cells. A The fabricating materials and processes. B The mechanism of killing tumor cells. C The catalytic activities of $\mathrm{MnO}_{2}$ @PtCo nanoflowers at different pH values. Reproduced with permission of Springer Nature (Wang et al. 2018)

A
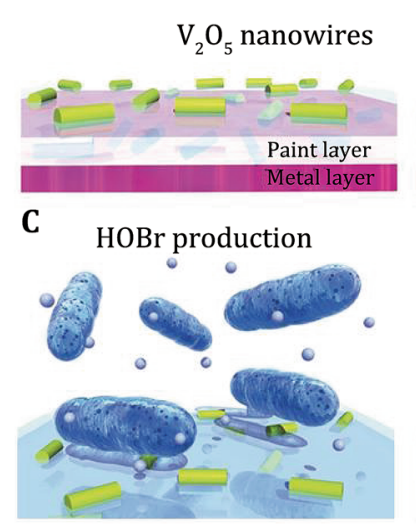

B Bacterial attack

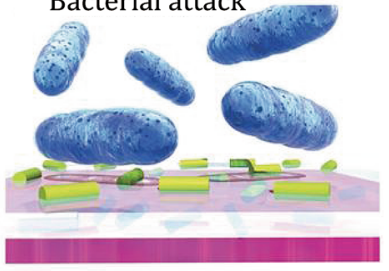

D

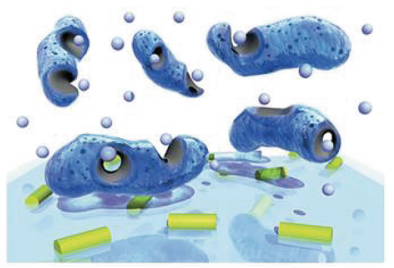

Fig. 16 Schemes of vanadium pentoxide nanozyme killing bacteria. A Nanozymes on a metal surface. B The bacteria attack. C The activities of nanozymes catalyze the generation of hypobromous acid. D The destruction of bacteria. Reproduced with permission of Springer Nature (Natalio et al. 2012)

catalase-like activities. With the capability of converting NIR light to generate ${ }^{1} \mathrm{O}_{2}$, the MOF nanozyme further killed the cancer cells (He et al. 2020). Glucoses were depleted by MOF nanozymes in tumor cells, leading to the loss of nutrition in these cancer cells. Meanwhile $\mathrm{H}_{2} \mathrm{O}_{2}$ was produced in this reaction and the catalase-like activity catalyzed $\mathrm{H}_{2} \mathrm{O}_{2}$ to generate $\mathrm{O}_{2}$. Upon NIR light irradiation, the nanozyme continuously converted the generated $\mathrm{O}_{2}$ to ${ }^{1} \mathrm{O}_{2}$, wounding the cells finally. This design demonstrated that MOF nanozyme is a promising

nanomaterial for multifunctional utilization, especially in vivo.

As for practical utilization, we will discuss a real smart biomedical machine for in vivo applications. This machine was not at nanoscale, but its function systems were built on nanomaterials (Son et al. 2015) (Fig. 17). The function systems were comprised of ceria nanozymes and gold nanorods decorated with mesoporous silica (AuNR@MSN). Ceria nanozymes with superoxide dismutatase activity could scavenge ROS to reduce inflammations and AuNR@MSN held a post of drug carrier. Thanks to NIR-responsive property, AuNR@MSN received wireless instructions by the photothermal signal, then the heat would result in the diffusion of drugs. Together with other systems: flow/temperature sensors and resistance random access memory (RRAM), the whole machine was an electronic stent for handling artery blockage. Ceria nanozymes induced in the novel stent could alleviate inflammations resulting from traditional mechanical stents.

Showing their capabilities in applications such as tumor therapy and antimicrobial utilization, nanozymes offer us a variety of ideas for building the function system. By selecting proper ones, we can endow the function system with multiplex functions. Especially, the nanozyme-based multifunctional nanoparticles may be utilized in the fabrication of nanomachines or nanorobots. At present, we are able to construct tiny smart machines like the electronic stent above, but we need to overcome the problems arousing in the manufacture of nanodevices such as the supply of power energy and the processing of instruction in the logic control system at nanoscale. 

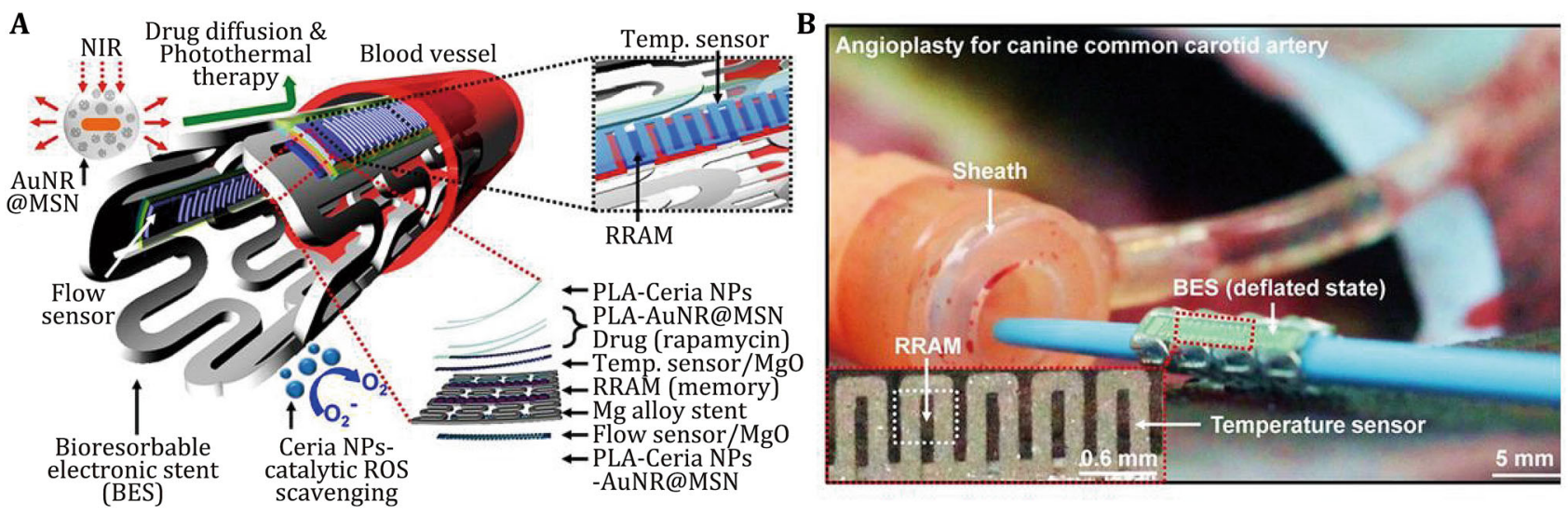

Fig. 17 The bioresorbable electronic stent. A The structure illustration. B The image of applications in vivo. Reproduced with permission of American Chemical Society (Son et al. 2015)

\section{NANOZYME AS THE BRIDGE FOR CONNECTION OF SYSTEMS}

Complicated functions and integrated systems should be combined together to fabricate nanorobots. However, the nanomachines which lack some partial components may limit the abilities of nanorobots. For instance, many functional nanoparticles lack a driving system (a motor), while some of the micro/nanomotors are not provided with function parts. In addition, the sensing system and logic control system are absent in a numerous of nanomachines. Therefore, the combination of properties of individual nanomachines will make a great contribution to build more powerful nanorobots. Nanozymes with multiple characteristics can be employed in various systems to connect different components, for their properties may simultaneously work in several systems.

Besides the enzyme-like activities, some characteristics of nanozymes make it possible to integrate separated systems, and $\mathrm{Fe}_{3} \mathrm{O}_{4}$ nanozyme is one of the examples. The magnetism of $\mathrm{Fe}_{3} \mathrm{O}_{4}$ nanozyme makes it a common component in the movement of the nanomachines. Trau's group made a platform for the isolation of in vitro circulating tumor cells (CTCs) that merely depended on the $\mathrm{Fe}_{3} \mathrm{O}_{4}$ nanozymes (Li et al. 2017). They linked $\mathrm{Fe}_{3} \mathrm{O}_{4}$ magnetic nanozymes $(30 \mathrm{~nm})$ with antibodies targeting melanoma cells (anti-MCSP). After incubation with cell suspensions, the $\mathrm{Fe}_{3} \mathrm{O}_{4}$ nanozymes attached to the melanoma CTC and the external magnetic field guided the isolation. The $\mathrm{Fe}_{3} \mathrm{O}_{4}$ nanozymes with separated CTCs would catalyze the colorimetric reaction based on the intrinsic peroxide-like activity (Fig. 18A). This method offers a rapid and convenient way to detect CTCs in vitro. Referring to the micro/nanomotor-based methods for isolation and the motion guidance of $\mathrm{Fe}_{3} \mathrm{O}_{4}$ nanozymes in driving system, this idea may provide an approach for the in vivo CTC detection, combining the function system with driving system.

Furthermore, gold nanozymes possess multiple characteristics as well. In one design, the CD-decorated gold nanozymes (CD@AuNPs) combined sensing, selfassembling and cascading enzyme-like catalysis together (Zhao et al. 2016). Au nanozymes accepted energy and participated in the fluorescence resonance energy transfer (FRET). After modified with cyclodextrin (CD), the new CD@AuNPs were appropriate for fluorescence sensing (Fig. 18B). Blood cholesterol assay was studied as the model, in which CD@AuNPs were added to rhodamine $\mathrm{B}(\mathrm{RB})$ molecules, and the combination of both materials led to the quenching-the sensing system was built. If the cholesterols were added to this sensing system, RB molecules would be taken place by cholesterol and dissociated from CD@AuNPs, back to the status of fluorescence recovery. This sensing system showed favorable results in the detection of blood cholesterol. Previous studies found that Au nanozymes exhibited glucose oxidase (GOx)-like activity, and some also possessed the peroxidase (HRP)-like activity. Here the CD@AuNPs with intrinsic GOx-like activity and HRP-like activity were able to catalyze cascade reactions, oxidizing glucose to generate $\mathrm{H}_{2} \mathrm{O}_{2}$ and catalyzing TMB (Fig. 18C). Finally, CD@AuNPs self-assembled into a well-ordered structure with tetrakis(4-carboxyphenyl)porphyrin (TCPP) acting as a mediator (Fig. 18D). Endowed with various properties, Au nanozymes are very likely to help the combination of the sensing and function systems. 

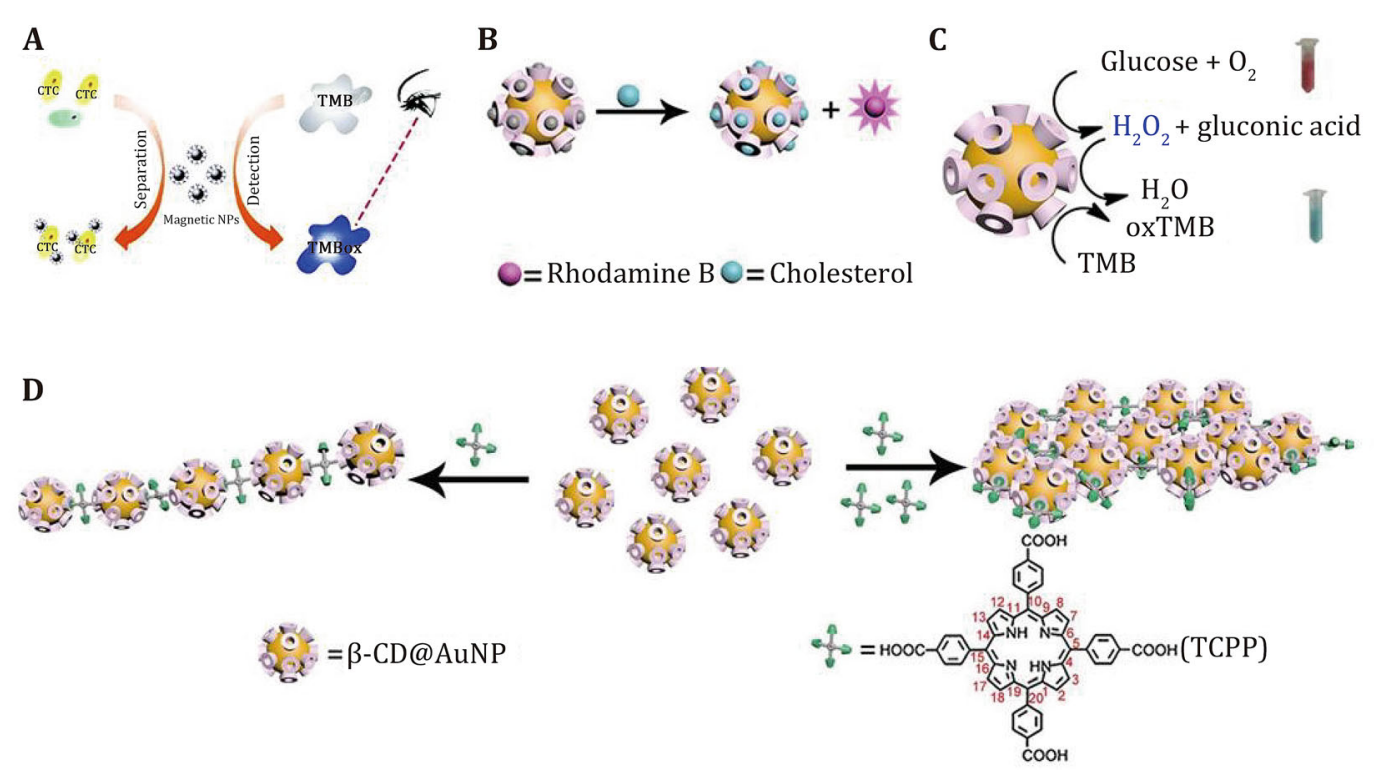

Fig. 18 A The detection of CTCs in vitro by $\mathrm{Fe}_{3} \mathrm{O}_{4}$ nanozymes. Reproduced with permission of Royal Society of Chemistry (Li et al. 2017). B The fluorescence-based sensing system of CD@AuNPs. C The enzyme-like activities of CD@AuNPs and the cascade reactions. D The selfassembly of CD@AuNPs through TCPP. Reproduced with permission of American Chemical Society (Zhao et al. 2016)

\section{CONCLUSION}

In this review, we have summarized recent progress on the nanozymes-based micro/nanodevices. Producing a smart machine at nanoscale demands well-performed nanomaterials, and nanozyme is one of the nanomaterials waiting to be exploited. The modulation of activities is proper for the construct of logic gates, and the catalytic activities of nanozymes are able to power the movement or help with the motion control. Other properties like magnetism may also be of good assistance. Nanozymes are easy to be modified with extra abilities, which are fit for the structure of the nanorobots as well. The multiple enzyme-like activities of one nanozyme will enhance the catalytic performance and favor the nanodevices with more than one function.

Some powerful nanomachines have already been built. To take a further step we should find methods to integrate these nanomachines to construct a whole multiplex nanorobot. Nanozymes with catalytic activities, physicochemical properties may be an important key for the combination of components. With high biocompatibility and low biotoxicity, nanozymes now have been widely used for clinical applications, which catalyze the $\mathrm{H}_{2} \mathrm{O}_{2}$ or glucose at the tumor sites. These molecules, especially high level of $\mathrm{H}_{2} \mathrm{O}_{2}$ act as fuels to power the micro/nanomotors. For example, hollow mesoporous organosilica nanoparticles (HMONs) decorated with catalase-like $\mathrm{MnO}_{\mathrm{x}}$ nanozymes were studied for the ultrasound (US)-triggered sonodynamic therapy (SDT) (Zhu et al. 2018). $\mathrm{MnO}_{\mathrm{x}}$ nanozymes decomposed
$\mathrm{H}_{2} \mathrm{O}_{2}$ in the tumor microenvironment (TME) to generate $\mathrm{O}_{2}$. These nanoparticles may be constructed to Janus particles-the $\mathrm{MnO}_{\mathrm{x}}$ nanozymes are only linked to the hemispherical surface, so the generated $\mathrm{O}_{2}$ could provide power for driving. In this way, the functional nanoparticles are able to help with the motion and sonodynamic therapy (SDT) simultaneously. More importantly, driving power may help the nanodevices go deep into the inner part of the tumor sites. We may need to break through the barrier that $\mathrm{H}_{2} \mathrm{O}_{2}$ in the TME is not enough to drive the movement currently. In addition, the TME offers modulations for chemical-responsive control, and problems may still remain in the complexity of the TME.

In conclusion, nanozymes are promising materials for the further fabrication of nanodevices such as nanomachines and nanorobots, and there are extensive opportunities for us to explore.

Acknowledgements This work was financially supported by the Strategic Priority Research Program of CAS (XDB29040101), the National Natural Science Foundation of China (31530026, 31871005, 31900981, 21907043), Chinese Academy of Sciences (YJKYYQ20180048), the Key Research Program of Frontier Sciences, CAS (QYZDY-SSW-SMC013), National Key Research and Development Program of China (2017YFA0205501) and Youth Innovation Promotion Association CAS (2019093).

\section{Compliance with Ethical Standards}

Conflict of interest Jiaying Xie, Yiliang Jin, Kelong Fan and Xiyun Yan declare that they have no conflict of interest. 
Human and animal rights and informed consent This article does not contain any studies with human or animal subjects performed by any of the authors.

Open Access This article is licensed under a Creative Commons Attribution 4.0 International License, which permits use, sharing, adaptation, distribution and reproduction in any medium or format, as long as you give appropriate credit to the original author(s) and the source, provide a link to the Creative Commons licence, and indicate if changes were made. The images or other third party material in this article are included in the article's Creative Commons licence, unless indicated otherwise in a credit line to the material. If material is not included in the article's Creative Commons licence and your intended use is not permitted by statutory regulation or exceeds the permitted use, you will need to obtain permission directly from the copyright holder. To view a copy of this licence, visit http://creativecommons.org/ licenses/by/4.0/.

\section{References}

Ayaz Ahmed KB, Subramanian S, Sivasubramanian A, Veerappan G, Veerappan A (2014) Preparation of gold nanoparticles using Salicornia brachiata plant extract and evaluation of catalytic and antibacterial activity. Spectrochimica Acta Part A 130:54-58

Balasubramanian S, Kagan D, Jack Hu C-M, Campuzano S, LoboCastañon MJ, Lim N, Kang DY, Zimmerman M, Zhang L, Wang J (2011) Micromachine-enabled capture and isolation of cancer cells in complex media. Angew Chem Int Ed 50(18):4161-4164

Baraban L, Harazim SM, Sanchez S, Schmidt OG (2013) Chemotactic behavior of catalytic motors in microfluidic channels. Angew Chem Int Ed Engl 52(21):5552-5556

Celikten A, Cetin A (2016) Recent advances, issues and patents on medical nanorobots. Recent Patents Eng 10(1):28-35

Choi H, Lee GH, Kim KS, Hahn SK (2018) Light-guided nanomotor systems for autonomous photothermal cancer therapy. ACS Appl Mater Interfaces 10(3):2338-2346

Das M, Patil S, Bhargava N, Kang JF, Riedel LM, Seal S, Hickman JJ (2007) Auto-catalytic ceria nanoparticles offer neuroprotection to adult rat spinal cord neurons. Biomaterials 28(10):1918-1925

Ding H, Cai Y, Gao L, Liang M, Miao B, Wu H, Liu Y, Xie N, Tang A, Fan K, Yan X, Nie G (2018) Exosome-like nanozyme vesicles for $\mathrm{H}_{2} \mathrm{O}_{2}$-responsive catalytic photoacoustic imaging of xenograft nasopharyngeal carcinoma. Nano Lett 19(1):203-209

Fan K, Cao C, Pan Y, Lu D, Yang D, Feng J, Song L, Liang M, Yan X (2012) Magnetoferritin nanoparticles for targeting and visualizing tumour tissues. Nat Nanotechnol 7(7):459-464

Fan K, Jia X, Zhou M, Wang K, Conde J, He J, Tian J, Yan X (2018a) Ferritin nanocarrier traverses the blood brain barrier and kills glioma. ACS Nano 12(5):4105-4115

Fan K, Xi J, Fan L, Wang P, Zhu C, Tang Y, Xu X, Liang M, Jiang B, Yan $\mathrm{X}$, Gao L (2018b) In vivo guiding nitrogen-doped carbon nanozyme for tumor catalytic therapy. Nat Commun 9(1):1440. https://doi.org/10.1038/s41467-018-03903-8

Fournier-Bidoz SB, Arsenault AC, Manners I, Ozin GA (2005) Synthetic self-propelled nanorotors. Chem Commun. https:// doi.org/10.1039/b414896g4
Gao L, Zhuang J, Nie L, Zhang J, Zhang Y, Gu N, Wang T, Feng J, Yang D, Perrett S, Yan X (2007) Intrinsic peroxidase-like activity of ferromagnetic nanoparticles. Nat Nanotechnol 2(9):577-583

Gao W, Uygun A, Wang J (2012) Hydrogen-bubble-propelled zincbased microrockets in strongly acidic media. J Am Chem Soc 134(2):897-900

Gao L, Giglio KM, Nelson JL, Sondermann H, Travis AJ (2014) Ferromagnetic nanoparticles with peroxidase-like activity enhance the cleavage of biological macromolecules for biofilm elimination. Nanoscale 6(5):2588-2593

Gao W, de Avila BE, Zhang L, Wang J (2018) Targeting and isolation of cancer cells using micro/nanomotors. Adv Drug Deliv Rev 125:94-101

Gao L, Gao X, Yan X (2020) Kinetics and mechanisms for nanozymes. In: Yan X et al. (eds) Kinetics and mechanisms for nanozymes. Springer, Singapore, pp 17-39

Gibbs JG, Zhao YP (2009) Autonomously motile catalytic nanomotors by bubble propulsion. Appl Phys Lett 94(16):163104. https://doi.org/10.1063/1.3122346

Guix M, Meyer AK, Koch B, Schmidt OG (2016) Carbonate-based Janus micromotors moving in ultra-light acidic environment generated by HeLa cells in situ. Sci Rep 6:21701. https://doi. org/10.1038/srep21701

Guo J, Gallegos JJ, Tom AR, Fan D (2018) Electric-field-guided precision manipulation of catalytic nanomotors for cargo delivery and powering nanoelectromechanical devices. ACS Nano 12(2):1179-1187

Guo Z, Wang T, Rawal A, Hou J, Cao Z, Zhang H, Xu J, Gu Z, Chen V, Liang K (2019) Biocatalytic self-propelled submarine-like metal-organic framework microparticles with pH-triggered buoyancy control for directional vertical motion. Mater Today 28:10-16

He Y, Wu J, Zhao Y (2007) Designing catalytic nanomotors by dynamic shadowing growth. Nano Lett 7(5):1369-1375

He X, Tan L, Chen D, Wu X, Ren X, Zhang Y, Meng X, Tang F (2013) $\mathrm{Fe}_{3} \mathrm{O}_{4}-\mathrm{Au} @$ mesoporous $\mathrm{SiO}_{2}$ microspheres: an ideal artificial enzymatic cascade system. Chem Commun 49(41):4643-4645

He L, Ni Q Mu J, Fan W, Liu L, Wang Z, Li L, Tang W, Liu Y, Cheng Y, Tang L, Yang Z, Liu Y, Zou J, Yang W, Jacobson O, Zhang F, Huang P, Chen X (2020) Solvent-assisted self-assembly of a metal-organic framework based biocatalyst for cascade reaction driven photodynamic therapy. J Am Chem Soc 142(14):6822-6832

Hong Y, Blackman NM, Kopp ND, Sen A, Velegol D (2007) Chemotaxis of nonbiological colloidal rods. Phys Rev Lett 99(17):178103. https://doi.org/10.1103/PhysRevLett.99. 178103

Hong Y, Velegol D, Chaturvedi N, Sen A (2010) Biomimetic behavior of synthetic particles: from microscopic randomness to macroscopic control. Phys Chem Chem Phys 12(7):1423-1435

Hortelão AC, Patiño T, Perez-Jiménez A, Blanco À, Sánchez S (2017) Enzyme-powered nanobots enhance anticancer drug delivery. Adv Funct Mater 28(25):1705086. https://doi.org/ 10.1002/adfm.201705086

Howse JR, Jones RA, Ryan AJ, Gough T, Vafabakhsh R, Golestanian R (2007) Self-motile colloidal particles: from directed propulsion to random walk. Phys Rev Lett 99(4):048102. https:// doi.org/10.1103/PhysRevLett.99.048102

Hu Q, Qian C, Sun W, Wang J, Chen Z, Bomba HN, Xin H, Shen Q Gu Z (2016) Engineered nanoplatelets for enhanced treatment of multiple myeloma and thrombus. Adv Mater 28(43):9573-9580

Huang Y, Zhao M, Han S, Lai Z, Yang J, Tan C, Ma Q, Lu Q, Chen J, Zhang X, Zhang Z, Li B, Chen B, Zong Y, Zhang H (2017) 
Growth of Au nanoparticles on 2D metalloporphyrinic metalorganic framework nanosheets used as biomimetic catalysts for cascade reactions. Adv Mater 29(32):1700102. https:// doi.org/10.1002/adma.201700102

Jiang B, Duan D, Gao L, Zhou M, Fan K, Tang Y, Xi J, Bi Y, Tong Z, Gao GF, Xie N, Tang A, Nie G, Liang M, Yan X (2018) Standardized assays for determining the catalytic activity and kinetics of peroxidase-like nanozymes. Nat Protoc 13(7):1506-1520

Jiang B, Yan L, Zhang J, Zhou M, Shi G, Tian X, Fan K, Hao C, Yan X (2019a) Biomineralization synthesis of the Cobalt nanozyme in SP94-ferritin nanocages for prognostic diagnosis of hepatocellular carcinoma. ACS Appl Mater Interfaces 11(10):9747-9755

Jiang D, Ni D, Rosenkrans ZT, Huang P, Yan X, Cai W (2019b) Nanozyme: new horizons for responsive biomedical applications. Chem Soc Rev 48(14):3683-3704

Kagan D, Calvo-Marzal P, Balasubramanian S, Sattayasamitsathit S, Manesh KM, Flechsig GU, Wang J (2009) Chemical sensing based on catalytic nanomotors: motion-based detection of trace silver. J Am Chem Soc 131(34):12082-12083

Khezri B, Beladi Mousavi SM, Krejčová L, Heger Z, Sofer Z, Pumera M (2019) Ultrafast electrochemical trigger drug delivery mechanism for nanographene micromachines. Adv Funct Mater 29(4):1806696. https://doi.org/10.1002/adfm. 201806696

Kim J-w, Dang CV (2006) Cancer's molecular sweet tooth and the warburg effect. Cancer Res 66(18):8927-8930

Kim DH, Cheang UK, Kőhidai L, Byun D, Kim MJ (2010) Artificial magnetotactic motion control of Tetrahymena pyriformis using ferromagnetic nanoparticles: a tool for fabrication of microbiorobots. Appl Phys Lett 97(17):173702. https://doi. org/10.1063/1.3497275

Kim K-W, Kim BC, Lee HJ, Kim J, Oh M-K (2011) Enzyme logic gates based on enzyme-coated carbon nanotubes. Electroanalysis 23(4):980-986

Kline TR, Paxton WF, Mallouk TE, Sen A (2005) Catalytic nanomotors: remote-controlled autonomous movement of striped metallic nanorods. Angew Chem Int Ed 44(5):744-746

Laocharoensuk R, Burdick J, Wang J (2008) Carbon-nanotubeinduced acceleration of catalytic nanomotors. ACS Nano 2(5):1069-1075

Li J, Sattayasamitsathit S, Dong R, Gao W, Tam R, Feng X, Ai S, Wang J (2014) Template electrosynthesis of tailored-made helical nanoswimmers. Nanoscale 6(16):9415-9420

Li J, Wang J, Wang Y, Trau M (2017) Simple and rapid colorimetric detection of melanoma circulating tumor cells using bifunctional magnetic nanoparticles. Analyst 142(24):4788-4793

Lien CW, Chen YC, Chang HT, Huang CC (2013) Logical regulation of the enzyme-like activity of gold nanoparticles by using heavy metal ions. Nanoscale 5(17):8227-8234

Lien CW, Tseng YT, Huang CC, Chang HT (2014) Logic control of enzyme-like gold nanoparticles for selective detection of lead and mercury ions. Anal Chem 86(4):2065-2072

Lin Y, Xu C, Ren J, Qu X (2012) Using thermally regenerable cerium oxide nanoparticles in biocomputing to perform label-free, resettable, and colorimetric logic operations. Angew Chem Int Ed Engl 51(50):12579-12583

Loukanov A, Gagov H, Nakabayashi S (2019a) Artificial nanomachines and nanorobotics. In: Mousa A et al. (eds) The road from nanomedicine to precision medicine. Jenny Stanford Publishing, Singapore, pp 515-532

Loukanov A, Nikolova S, Filipov C, Nakabayashi S (2019b) Nanomaterials for cancer medication: from individual nanoparticles toward nanomachines and nanorobots. Pharmacia 66(3):147-156
Ma X, Jang S, Popescu MN, Uspal WE, Miguel-López A, Hahn K, Kim D-P, Sánchez S (2016) Reversed janus micro/nanomotors with internal chemical engine. ACS Nano 10(9):8751-8759

Manesh KM, Campuzano S, Gao W, Lobo-Castañón MJ, Shitanda I, Kiantaj K, Wang J (2013) Nanomotor-based biocatalytic patterning of helical metal microstructures. Nanoscale 5(4):1310-1314

Mano N, Heller A (2005) Bioelectrochemical propulsion. J Am Chem Soc 127(33):11574-11575

Masadeh MM, Karasneh GA, Al-Akhras MA, Albiss BA, Aljarah KM, Al-azzam SI, Alzoubi KH (2015) Cerium oxide and iron oxide nanoparticles abolish the antibacterial activity of ciprofloxacin against gram positive and gram negative biofilm bacteria. Cytotechnology 67(3):427-435

Mirkovic T, Zacharia NS, Scholes GD, Ozin GA (2010) Fuel for thought: chemically powered nanomotors out-swim nature's flagellated bacteria. ACS Nano 4(4):1782-1789

Mohamed MM, Fouad SA, Elshoky HA, Mohammed GM, Salaheldin TA (2017) Antibacterial effect of gold nanoparticles against Corynebacterium pseudotuberculosis. Int J Vet Sci Med 5(1):23-29

Munir S, Shah AA, Rahman H, Bilal M, Rajoka MSR, Khan AA, Khurshid M (2020) Nanozymes for medical biotechnology and its potential applications in biosensing and nanotherapeutics. Biotechnol Lett 42(3):357-373

Natalio F, Andre R, Hartog AF, Stoll B, Jochum KP, Wever R, Tremel W (2012) Vanadium pentoxide nanoparticles mimic vanadium haloperoxidases and thwart biofilm formation. Nat Nanotechnol 7(8):530-535

Paxton WF, Kistler KC, Olmeda CC, Sen A, St Angelo SK, Cao Y, Mallouk TE, Lammert PE, Crespi VH (2004) Catalytic nanomotors: autonomous movement of striped nanorods. J Am Chem Soc 126(41):13424-13431

Perez JM, Asati A, Nath S, Kaittanis C (2008) Synthesis of biocompatible dextran-coated nanoceria with $\mathrm{pH}$-dependent antioxidant properties. Small 4(5):552-556

Qin W, Peng T, Gao Y, Wang F, Hu X, Wang K, Shi J, Li D, Ren J, Fan C (2017) Catalysis-driven self-thermophoresis of Janus plasmonic nanomotors. Angew Chem Int Ed 56(2):515-518

Sharma V, Mobin SM (2017) Cytocompatible peroxidase mimic $\mathrm{CuO}$ :graphene nanosphere composite as colorimetric dual sensor for hydrogen peroxide and cholesterol with its logic gate implementation. Sens Actuators B 240:338-348

Sierra DP, Weir NA, Jones JF (2005) A review of research in the field of nanorobotics (No. SAND2005-6808). Sandia National Laboratories

Singh S, Dosani T, Karakoti AS, Kumar A, Seal S, Self WT (2011) A phosphate-dependent shift in redox state of cerium oxide nanoparticles and its effects on catalytic properties. Biomaterials 32(28):6745-6753

Son D, Lee J, Lee DJ, Ghaffari R, Yun S, Kim SJ, Lee JE, Cho HR, Yoon S, Yang S, Lee S, Qiao S, Ling D, Shin S, Song JK, Kim J, Kim T, Lee H, Kim J, Soh M, Lee N, Hwang CS, Nam S, Lu N, Hyeon T, Choi SH, Kim DH (2015) Bioresorbable electronic stent integrated with therapeutic nanoparticles for endovascular diseases. ACS Nano 9(6):5937-5946

Szatrowski TP, Nathan CF (1991) Production of large amounts of hydrogen peroxide by human tumor cells. Cancer Res 51(3):794-798

Tregubov AA, Nikitin PI, Nikitin MP (2018) Advanced smart nanomaterials with integrated logic-gating and biocomputing: dawn of theranostic nanorobots. Chem Rev 118(20):10294-10348

Van Haastert PJ, Devreotes PN (2004) Chemotaxis: signalling the way forward. Nat Rev Mol Cell Biol 5(8):626-634 
Vaupel P (2004) Tumor microenvironmental physiology and its implications for radiation oncology. Semin Radiat Oncol 14:198-206

Vicario J, Eelkema R, Browne WR, Meetsma A, La Crois RM, Feringa BL (2005) Catalytic molecular motors: fuelling autonomous movement by a surface bound synthetic manganese catalase. Chem Commun 31:3936-3938

Villa K, Krejčová L, Novotný F, Heger Z, Sofer Z, Pumera M (2018) Cooperative multifunctional self-propelled paramagnetic microrobots with chemical handles for cell manipulation and drug delivery. Adv Funct Mater 28(43):1804343. https:// doi.org/10.1002/adfm.201804343

Wan M, Chen H, Wang Q Niu Q, Xu P, Yu Y, Zhu T, Mao C, Shen J (2019) Bio-inspired nitric-oxide-driven nanomotor. Nat Commun 10(1):966. https://doi.org/10.1038/s41467-01908670-8

Wang J, Manesh KM (2010) Motion control at the nanoscale. Small 6(3):338-345

Wang H, Pumera M (2015) Fabrication of micro/nanoscale motors. Chem Rev 115(16):8704-8735

Wang C-I, Huang C-C, Lin Y-W, Chen W-T, Chang H-T (2012) Catalytic gold nanoparticles for fluorescent detection of mercury(II) and lead(II) ions. Anal Chim Acta 745:124-130

Wang J, Xiong Z, Zhan X, Dai B, Zheng J, Liu J, Tang J (2017a) A silicon nanowire as a spectrally tunable light-driven nanomotor. Adv Mater 29(30):1701451. https://doi.org/10.1002/ adma.201701451

Wang Z, Dong K, Liu Z, Zhang Y, Chen Z, Sun H, Ren J, Qu X (2017b) Activation of biologically relevant levels of reactive oxygen species by $\mathrm{Au} / \mathrm{g}-\mathrm{C} 3 \mathrm{~N} 4$ hybrid nanozyme for bacteria killing and wound disinfection. Biomaterials 113:145-157

Wang Z, Zhang Y, Ju E, Liu Z, Cao F, Chen Z, Ren J, Qu X (2018) Biomimetic nanoflowers by self-assembly of nanozymes to induce intracellular oxidative damage against hypoxic tumors. Nat Commun 9(1):3334. https://doi.org/10.1038/ s41467-018-05798-x

Wang J, Dong R, Wu H, Cai Y, Ren B (2019) A review on artificial micro/nanomotors for cancer-targeted delivery, diagnosis, and therapy. Nano-Micro Lett 12(1):1-19. https://doi.org/10. 1007/s40820-019-0350-5
Xu B, Zhang B, Wang L, Huang G, Mei Y (2018) Tubular micro/nanomachines: from the basics to recent advances. Adv Funct Mater 28(25):1705872. https://doi.org/10.1002/ adfm.201705872

Xu Y, Fei J, Li G, Yuan T, Xu X, Li J (2019) Nanozyme-catalyzed cascade reactions for mitochondria-mimicking oxidative phosphorylation. Angew Chem Int Ed 58(17):5572-5576

Yan X, Gao L (2020) Nanozymology: An Overview. In: Yan X et al. (eds) Nanozymology: an overview. Springer, Singapore, pp 3-16

Yang X, Yang J, Wang L, Ran B, Jia Y, Zhang L, Yang G, Shao H, Jiang $X$ (2017) Pharmaceutical intermediate-modified gold nanoparticles: against multidrug-resistant bacteria and wound-healing application via an electrospun scaffold. ACS Nano 11(6):5737-5745

Yang J, Zhang C, Wang X, Wang W, Xi N, Liu L (2018) Development of micro- and nanorobotics: a review. Sci China Technol Sci 62(1):1-20

Yoshida W, Yokobayashi Y (2007) Photonic Boolean logic gates based on DNA aptamers. Chem Commun. https://doi.org/10. 1039/b613201d2):195-197

Zha F, Wang T, Luo M, Guan J (2018) Tubular micro/nanomotors: propulsion mechanisms, fabrication techniques and applications. Micromachines 9(2):78. https://doi.org/10.3390/ $\operatorname{mi} 9020078$

Zhang X, Chen C, Wu J, Ju H (2019) Bubble-propelled jellyfish-like micromotors for DNA sensing. ACS Appl Mater Interfaces 11(14):13581-13588

Zhang Y, Jin Y, Cui H, Yan X, Fan K (2020) Nanozyme-based catalytic theranostics. RSC Adv 10(1):10-20

Zhao Y, Huang Y, Zhu H, Zhu Q Xia Y (2016) Three-in-one: sensing, self-assembly, and cascade catalysis of cyclodextrin modified gold nanoparticles. J Am Chem Soc 138(51):16645-16654

Zhou D, Ren L, Li YC, Xu P, Gao Y, Zhang G, Wang W, Mallouk TE, Li L (2017) Visible light-driven, magnetically steerable gold/ iron oxide nanomotors. Chem Commun 53(83):11465-11468

Zhu P, Chen Y, Shi J (2018) Nanoenzyme-augmented cancer sonodynamic therapy by catalytic tumor oxygenation. ACS Nano 12(4):3780-3795 